\title{
Genome-Wide Investigation and Expression Profiling of HD-Zip Transcription Factors in Foxtail Millet (Setaria italica L.)
}

\author{
Wenbo Chai, Weina Si, Wei Ji, Qianqian Qin, Manli Zhao, and Haiyang Jiang $\mathbb{E}$ \\ National Engineering Laboratory of Crop Stress Resistance Breeding, Anhui Agricultural University, Hefei 230036, China \\ Correspondence should be addressed to Haiyang Jiang; hyjiangahau@163.com
}

Received 6 July 2017; Revised 15 February 2018; Accepted 7 March 2018; Published 15 May 2018

Academic Editor: Yong-Pyo Lim

Copyright (C) 2018 Wenbo Chai et al. This is an open access article distributed under the Creative Commons Attribution License, which permits unrestricted use, distribution, and reproduction in any medium, provided the original work is properly cited.

\begin{abstract}
HD-Zip proteins represent the major transcription factors in higher plants, playing essential roles in plant development and stress responses. Foxtail millet is a crop to investigate the systems biology of millet and biofuel grasses and the HD-Zip gene family has not been studied in foxtail millet. For further investigation of the expression profile of the HD-Zip gene family in foxtail millet, a comprehensive genome-wide expression analysis was conducted in this study. We found 47 protein-encoding genes in foxtail millet using BLAST search tools; the putative proteins were classified into four subfamilies, namely, subfamilies I, II, III, and IV. Gene structure and motif analysis indicate that the genes in one subfamily were conserved. Promotor analysis showed that HD-Zip gene was involved in abiotic stress. Duplication analysis revealed that $8(\sim 17 \%)$ hdz genes were tandemly duplicated and 28 (58\%) were segmentally duplicated; purifying duplication plays important roles in gene expansion. Microsynteny analysis revealed the maximum relationship in foxtail millet-sorghum and foxtail millet-rice. Expression profiling upon the abiotic stresses of drought and high salinity and the biotic stress of ABA revealed that some genes regulated responses to drought and salinity stresses via an ABA-dependent process, especially sihdz29 and sihdz45. Our study provides new insight into evolutionary and functional analyses of HD-Zip genes involved in environmental stress responses in foxtail millet.
\end{abstract}

\section{Introduction}

The homeodomain leucine zipper (HD-Zip) gene family is a major group of transcription factors in the higher plants, which include a DNA-binding domain (HD) at the Cterminal and a leucine-zipper domain (bZIP also known as LZ domain) that can interact with other proteins $[1,2]$. Through the leucine-zipper domain, HD-Zip proteins fold into homo- or heterodimers, which is necessary for DNAbinding specificity $[3,4]$. Moreover, plants with an HD-Zip transgene exhibited improved morphological features when subjected to different stressful environmental conditions. The HD-Zip gene family can be divided into four classes (HD-Zip I-IV) according to their sequence similarity and structures of their different domains [5, 6]. A structural comparison found that all four classes contained the conserved HD and LZ domains, which promote dimerization, a prerequisite for DNA binding [6]. A minor distinction among the groups was also found; namely, HD-Zip I and HD-Zip
II can recognize the pseudopalindromic DNA sequence CAAT(A/T)ATTG [3, 7], whereas HD-Zip III and HD-Zip IV recognize GTAAT(G/C)ATTAC and TAAATG(C/T)A, respectively $[8,9]$. Furthermore, HD-Zip II contains two additional sets of five conserved amino acids, Cys, Pro, Ser, Cys, and Glu, known as CPSCG, and N-terminal consensus sequences. HD-Zip III and HD-Zip IV share a highly conserved lipid/sterol binding region called the START domain, followed by the start adjacent domain (SAD) [10, 11]. In addition, HD-Zip III proteins have the DNA-interacting PAS-related MEMKHLA domain at their C-terminus [12]. The expression level of HD-Zip IV is associated with the epidermis in general or specifically with its structure [13].

To date, HD-Zip-encoding genes have been analyzed in several plants, such as arabidopsis (Arabidopsis thaliana) [14], maize (Zea mays) [15], rice (Oryza sativus) [16], poplar (Populus trichocarpa) [17], cucumber (Cucumis sativus) [18], soybean (Glycine max) [19], and chrysanthemum (Chrysanthemum morifolium) [20]. Genes have different domains 
that enable them to participate in very diverse biological processes, but genes within the same family tend to have some functional conservation [6]. HD-Zip I proteins play important roles in plant responses to various environmental conditions, such as drought, exposure to abscisic acid (ABA), salinity stress, and extreme temperature. The oshox 22 gene belongs to HD-Zip I, the expression of which is strongly induced by salt stress, $\mathrm{ABA}$, and polyethylene glycol (PEG) treatment, and weakly induced by cold stress [21]. HB1 in Medicago truncatula participates in responses to salt and osmotic stresses and is expressed in primary and lateral root meristem [22]. Zhao et al. also found that HD-Zip I genes were regulated by drought stress [15]. More recently, the maize HD-Zip I gene Zmhdz10 was reported to positively regulate drought and salt tolerance in plants through an $\mathrm{ABA}$ dependent signaling pathway [23]. In the case of HD-Zip II proteins, they are mainly involved in shade avoidance or light signaling, plant development, and abiotic stress tolerance [7]. For example, the expression of ATHB2, HAT2, HAT3, and HAT4 is regulated by phytochrome, in that a low red/farred light ratio rapidly induces the shade-avoidance response $[24,25]$. Furthermore, ATHB4 and HAT3 play critical roles in establishing bilateral symmetry in cotyledons and developing leaves $[25,26]$. HAHB10, a sunflower HD-Zip II transcription factor, has also been shown to participate in the transition from the vegetative to the flowering stage and to mediate the content of phytohormones upon biotic stress [27].

HD-Zip III proteins were reported to act as regulators that participate in axial cell elongation, xylem differentiation, auxin transport, and lateral organ initiation [28, 29]. ZeHB13, a HD-Zip III gene in Zinnia, is a pivotal transcriptional regulator responsible for early vascular development [30]. Arabidopsis REV (REVOLUTA) can regulate meristem initiation at a lateral position [31]. In the case of HD-Zip IV proteins, these play crucial roles in epidermal development [32]; these proteins are thought to participate in trichome formation, lipid metabolism, and cuticle biosynthesis [33]. Maize OCL4 (outer cell layer 4) inhibits the patterning of the trichome and influences the division or differentiation of the anther [34].

Foxtail millet is a cultivated form of Setaria, the wild ancestor of which is green foxtail $[35,36]$. Setaria is an important food and biofuel in northern China $[37,38]$. It is important to improve its product, which is associated with the environment. HD-Zip transcription factors can improve the morphological features upon exposure to stress. The key characteristics of Setaria are its small genome size, short life cycle, and close resemblance to maize and sorghum $[39,40]$. High-quality sequencing of Setaria has been completed by Bennetzen et al. and Wang et al. [41, 42], which provided the foundation for genome-wide analysis of the HD-Zip genes. In this study, we found 47 protein-encoding genes in foxtail millet and 25 genes in green foxtail using BLAST search tools; we found that the putative proteins could be classified into four subfamilies. Structural and motif analyses were used to identify the characteristics of the HD-Zip genes. Then, Blast2GO, duplication, and microsynteny analyses were performed to predict the function of the genes in foxtail millet. To further identify the functions of the HD-Zip genes, expression profiling upon exposure to abiotic stresses such as drought and high salinity, and the biotic stress, ABA was performed. Overall, our results provide a foundation for further study of the functions of HD-Zip genes, particularly for members with potentially important functions in the responses to abiotic stresses, for application in molecular breeding.

\section{Materials and Methods}

2.1. Sequence Retrieval and Identification of HD-Zip Genes. The Hidden Markov Model (HMM) of Homeobox-associated leucine zipper (PF02183) was used to identify the foxtail millet HD-Zip genes in a protein database with the BLASTP program $(P$ value $=0.001)$. Then, the obtained proteins were examined by Pfam (http://pfam.xfam.org/) [43] and SMART (http://smart.embl-heidelberg.de/) [44] for the presence of $\mathrm{HD}$ and Zip domains. The sequence IDs, protein sequences, genomic sequences, and CDS sequences were obtained from Phytozome (http://www.phytozome.net). Information about the physical parameters of the predicted HD-Zip proteins was obtained from the online ExPASy programs (http://web .expasy.org/protparam/), including the length of proteins, molecular weight $(\mathrm{kDa})$, and isoelectric point.

2.2. Chromosomal Location, Gene Organization, and Conserved Motif Analyses. Chromosome information was obtained from Phytozome (http://www.phytozome.net). The chromosomal location map of the genes was generated by MapInspect (http://mapinspect.software.informer.com) and the genes were renamed according to their initial position on the chromosomes. To determine the gene structure, the intron-exon organization was analyzed by Gene Structure Display Server (GSDS; http://gsds.cbi.pku.edu.cn) via alignment of the CDS sequences with their corresponding genomic sequences. The online MEME program was used to perform the structure of motif (http://meme-suite.org/tools/ meme) [45], using the default parameters. In addition, structure motif annotation was performed by Pfam and SMART.

2.3. Ka/Ks Values. Protein sequences of duplicated pairs were firstly aligned by MEGA v7.0 and then aligned sequences were used to calculate values of Ka/Ks by DnaSP v6.0. The Ks value was translated into duplication time in millions of years based on a rate of $\lambda$ substitutions per synonymous site per year. The duplication time of duplicated genes was calculated by $T=$ $\mathrm{Ks} / 2 \lambda \times 10^{-6} \mathrm{Mya}\left(\lambda=6.5 \times 10^{-9}\right.$ for grasses $)[10]$.

2.4. Phylogenetic Analysis, Gene Ontology (GO) Annotation, and Promoter Analysis. The protein sequences of HD-Zip were used to construct the phylogenetic tree with MEGA 7.0, using the neighbor-joining (NJ) method with 1000 bootstrap replications [46]. The GO annotation of the HD-Zip protein sequences was performed using Blast2GO (http://www .blast2go.com) [47], and the functions were defined into three categories: biological processes, cellular components, and 
molecular functions. The sequences about 2000 bp upstream of the transcription start site (ATG) were acquired using Phytozome. The online database PLACE (http://www.dna .affrc.go.jp/PLACE/signalscan.html) [48] was also employed to investigate putative cis-regulatory elements.

2.5. Modeling Construct of HD-Zip Proteins and Evolutionary Analysis of Paralogous and Orthologous Genes. The three-dimensional structures of HD-Zip proteins were used by Phyre2 server (Protein Homology/AnalogY Recognition Engine; http://www.sbg.bio.ic.ac.uk/phyre2) [49]. The position of the HD-Zip genes was marked by Perl script. The orthologous NAT genes among foxtail millet, maize, rice, sorghum, Brachypodium distachyon, green foxtail, and switchgrass were identified using OrthoMCL (http:// orthomcl.org/orthomcl/) [50]. Then, the similarity of the orthologous genes was rechecked using local blast. The relationships of the orthologous pairs among the six species were plotted using Circos (http://circos.ca/) [51].

2.6. Expression Profiling of Tissues. To gain insight into the tissue-specific gene expression patterns of HD-Zip genes, the Setaria italica Illumina RNA-HiSeq reads for four tissues were retrieved from the European Nucleotide Archive [SRX128226 (spica), SRX128225 (stem), SRX128224 (leaf), and SRX128223 (root)]. Then, the low-quality reads were removed using the NGS toolkit (http://www.nipgr.res.in/ngsqctoolkit.html). The reads per kilobase per million (RPKM) values were used to normalize the mapped reads. A heat map was generated based on the RPKM values for each gene in all tissue samples using RNA-Seq data.

2.7. Plant Growth and Stress Treatment. The cultivar Yugul seedlings of foxtail millet were grown in a growth chamber under the following conditions: $28 \pm 2^{\circ} \mathrm{C}$ with a 14-hour light/10-hour dark photoperiod. Different treatments were applied to 3-week-old plants. ABA stress was conducted by spreading $20 \mu \mathrm{M}$ ABA on the plants, and the plant leaves were harvested at $0,1,3,6,12$, and $24 \mathrm{~h}$ after this treatment. For drought stress and salt stress, 25\% PEG 6000 (dehydration) and $250 \mathrm{mM} \mathrm{NaCl}$ were used to water the plants, and the leaves were collected at $0,1,3,6,12$, and $24 \mathrm{~h}$. The samples were immediately frozen in liquid nitrogen and stored at $-80^{\circ} \mathrm{C}$ until RNA extraction. Three biological replications were conducted per sample.

2.8. RNA Extraction and Quantitative Real-Time PCR Analysis. Total RNA was isolated following the method described by Huang et al. [52]. The quality and purity were assessed by $1.2 \%$ agarose gel and a NanoDrop ND-1000 spectrophotometer. The first-strand cDNAs were synthesized using RTPCR Quick Master Mix (PCR-301; TOYOBO). The primers were designed by Primer3, and the NCBI database was used to identify their specificity. A constitutive Act2 gene-based primer was used as an endogenous control. The expression level was analyzed by the $2^{-\Delta \Delta C t}$ method and the picture was drawn using SigmaPlot 10.0.

\section{Results}

3.1. Identification of the HD-Zip Genes in Foxtail Millet. Highquality sequencing has been completed in many species, which provides a foundation to study the HD-Zip gene family. The HMM sequence was used here to search for the foxtail millet HD-Zip genes in Phytozome. First, a total of 52 genes were found, after which all genes were confirmed by Pfam and SMART. By removing the different transcripts of the same gene, 47 nonredundant genes were identified in foxtail millet, which were named sihdzl-sihdz47 based on their physical locations on chromosomes 1-9 (Table 1). The proteins have diverse lengths from 220 to 871 amino acids (aa), with an average of 506 aa. Moreover, the molecular weight ranged from 24.2 to $93.2 \mathrm{kDa}$ and the isoelectric point varied from 4.6 to 9.63. All information on sihdz protein sequences is summarized in Table 1 .

3.2. Phylogenetic Classification and Structure of HD-Zip in Foxtail Millet. To classify the HD-Zip genes in foxtail millet, the protein sequences in maize (54), rice (47), sorghum (41), and green foxtail (25) were all searched to generate an unrooted phylogenetic tree (Figure 1). Based on high bootstrap values, HD-Zip genes were divided into four subfamilies, I-IV. Subfamily III has the fewest HD-Zip genes, namely, only 22, while subfamily I has 68 genes, subfamily II has 67 genes, and subfamily IV has 57 genes.

To further investigate the evolutionary relationships among the $47 \mathrm{HD}$-Zip proteins in foxtail millet and 25 proteins in green foxtail, an unrooted phylogenetic tree was constructed by the NJ method, with 1000 bootstrap replications (Figure 2(a)). The HD-Zip genes were distinctly divided into the four subfamilies. In foxtail millet, subfamily IV had the highest number of members (16), followed by subfamily I and subfamily II, having the same number of members (13 each), while subfamily III had the lowest number of members. Interestingly, the green foxtail genes only clustered in subfamily I and subfamily II. Phylogenetic analysis revealed the presence of 18 gene pairs in foxtail millet, with strong bootstrap values (>91\%).

The CDSs and their corresponding DNA sequences were used to investigate the structure of HD-Zip genes. The results indicate that the intron-exon structure conformed to the results of phylogenetic analysis (Figure 2(b)). Genes in each subfamily were shown to have very similar numbers of introns, such as subfamily I and subfamily II, each having from 1 to 3 introns, subfamily III having the most introns at 16-17, and subfamily IV having between 6 and 10 .

The MEME online tool was used to identify the conserved motifs. A total of 25 motifs were investigated; the details of motif length and sequence are shown in Table 2. Every putative motif was annotated using Pfam and SMART. For simplicity, motifs that were not annotated are not shown in Figure 2. The results divided the HD-Zip gene family into four subfamilies, which were completely consistent with the classification from the phylogenetic analysis. Among these, motif 1 and motif 2 were found to encode the HD domain, and motif 4 and motif 18 were found to encode the LZ domain. The HD domain (motif 1 and motif 2 ) was found to be present 
TABLE 1: Feature list of 47 genes identified in foxtail millet and their sequence characteristics.

\begin{tabular}{|c|c|c|c|c|c|c|c|c|c|}
\hline \multirow[b]{2}{*}{ NIPGR ID } & \multirow[b]{2}{*}{$\begin{array}{l}\text { Phytozome } \\
\text { Identifier }\end{array}$} & \multicolumn{3}{|c|}{ Physical position on foxtail millet genome } & \multirow[b]{2}{*}{$\begin{array}{c}\text { Protein } \\
\text { length (aa) }\end{array}$} & \multirow[b]{2}{*}{ PI } & \multirow{2}{*}{$\begin{array}{c}\text { Molecular } \\
\text { weight } \\
\text { (Da) }\end{array}$} & \multirow[b]{2}{*}{ Intron } & \multirow[b]{2}{*}{$\begin{array}{c}\text { Phylogeny } \\
\text { group }\end{array}$} \\
\hline & & Chromosome & $\begin{array}{l}\text { Start position } \\
\text { (bp) }\end{array}$ & $\begin{array}{l}\text { End position } \\
\text { (bp) }\end{array}$ & & & & & \\
\hline Sihdz01 & Seita.1G087900 & 1 & 7798792 & 7799706 & 223 & 9.63 & 24184.04 & 1 & I \\
\hline Sihdz02 & Seita.1G088000 & 1 & 7808171 & 7809619 & 220 & 9.58 & 23994.74 & 1 & I \\
\hline Sihdz03 & Seita.1G201700 & 1 & 28159965 & 28165140 & 327 & 6.1 & 36098.44 & 3 & $\mathrm{I}$ \\
\hline Sihdz04 & Seita.1G253700 & 1 & 32918259 & 32922133 & 236 & 5.4 & 26468.23 & 1 & II \\
\hline Sihdz05 & Seita.1G306100 & 1 & 36983614 & 36985721 & 324 & 4.6 & 35439.06 & 3 & II \\
\hline Sihdz06 & Seita.1G335800 & 1 & 39351189 & 39355603 & 711 & 6.2 & 77892.71 & 9 & IV \\
\hline Sihdz07 & Seita.2G112400 & 2 & 10947386 & 10950661 & 735 & 6.4 & 79319.1 & 7 & IV \\
\hline Sihdz08 & Seita.2G114000 & 2 & 11221021 & 11225092 & 712 & 6.21 & 78529.74 & 7 & IV \\
\hline Sihdz09 & Seita.2G193200 & 2 & 28965749 & 28968902 & 303 & 4.65 & 32897.23 & 2 & II \\
\hline \multirow{3}{*}{ Sihdz10 } & Seita.2G224000 & 2 & 32601420 & 32603671 & 352 & 6.06 & 36851.92 & 3 & I \\
\hline & Seita.2G224000.2 & 2 & 32601420 & 32603671 & 419 & 9.69 & 43426.69 & & \\
\hline & Seita.2G224000.3 & 2 & 32601420 & 32603671 & 313 & 9.32 & 33026.8 & & \\
\hline Sihdz11 & Seita.2G236800 & 2 & 34125151 & 34127695 & 265 & 4.82 & 28604.67 & 2 & II \\
\hline Sihdz12 & Seita.2G282200 & 2 & 37895221 & 37901754 & 860 & 6.17 & 91157.98 & 8 & IV \\
\hline Sihdz13 & Seita.2G284000 & 2 & 38029446 & 38031046 & 237 & 5.3 & 26082.92 & 1 & II \\
\hline Sihdz14 & Seita.2G368600 & 2 & 44278030 & 44279425 & 230 & 6.02 & 25541.64 & 2 & II \\
\hline Sihdz15 & Seita.3G395000 & 3 & 49640251 & 49646758 & 853 & 6.37 & 92896.04 & 17 & III \\
\hline Sihdz16 & Seita.4G029000 & 4 & 1875175 & 1876650 & 271 & 9.4 & 28195.86 & 1 & $\mathrm{I}$ \\
\hline Sihdz17 & Seita.4G029200 & 4 & 1896185 & 1897677 & 291 & 9.15 & 30526.21 & 1 & $\mathrm{I}$ \\
\hline Sihdz18 & Seita.4G084600 & 4 & 6822689 & 6826925 & 699 & 5.89 & 75992.54 & 9 & IV \\
\hline Sihdz19 & Seita.4G245400 & 4 & 36871689 & 36872853 & 223 & 9.28 & 24394.21 & 2 & I \\
\hline Sihdz20 & Seita.5G079000 & 5 & 6743029 & 6750110 & 806 & 5.3 & 86426.43 & 8 & IV \\
\hline Sihdz21 & Seita.5G141300 & 5 & 12454752 & 12459714 & 855 & 5.56 & 92530.12 & 16 & III \\
\hline Sihdz22 & Seita.5G241600 & 5 & 30455176 & 30458970 & 224 & 9.35 & 24654.92 & 3 & $\mathrm{I}$ \\
\hline Sihdz23 & Seita.5G326100 & 5 & 37528162 & 37531618 & 800 & 6.3 & 85890.61 & 8 & IV \\
\hline Sihdz24 & Seita.5G344800 & 5 & 38943201 & 38948786 & 710 & 8.5 & 78461.92 & 10 & IV \\
\hline Sihdz25 & Seita.6G039900 & 6 & 3018210 & 3023859 & 785 & 5.69 & 84606.48 & 9 & IV \\
\hline Sihdz26 & Seita.6G072700 & 6 & 6484850 & 6490160 & 793 & 5.6 & 85138.95 & 6 & IV \\
\hline Sihdz27 & Seita.6G145000 & 6 & 25873860 & 25878078 & 280 & 4.81 & 30601.69 & 2 & II \\
\hline \multirow{2}{*}{ Sihdz28 } & Seita.6G176400 & 6 & 30029766 & 30031922 & 355 & 7.06 & 36870.92 & 3 & I \\
\hline & Seita.6G176400.2 & 6 & 30029766 & 30031922 & 353 & 6.68 & 36614.61 & & \\
\hline Sihdz29 & Seita.6G187700 & 6 & 31175081 & 31177598 & 279 & 4.83 & 29580.76 & 3 & II \\
\hline Sihdz30 & Seita.7G060900 & 7 & 16153965 & 16157882 & 715 & 6.07 & 78947.15 & 6 & IV \\
\hline Sihdz31 & Seita.7G061100 & 7 & 16176368 & 16179683 & 730 & 6.01 & 80631.47 & 7 & IV \\
\hline Sihdz32 & Seita.7G063300 & 7 & 16569209 & 16575494 & 674 & 6.3 & 74387.26 & 8 & IV \\
\hline Sihdz33 & Seita.7G184600 & 7 & 26206329 & 26207830 & 335 & 6.6 & 37451.98 & 2 & II \\
\hline Sihdz34 & Seita.7G187800 & 7 & 26435171 & 26436840 & 237 & 9.06 & 26043.72 & 3 & $\mathrm{I}$ \\
\hline Sihdz35 & Seita.7G203300 & 7 & 27549926 & 27555890 & 793 & 5.87 & 85333.81 & 8 & IV \\
\hline Sihdz36 & Seita.7G246700 & 7 & 30566314 & 30572371 & 781 & 5.48 & 84480.42 & 9 & IV \\
\hline Sihdz37 & Seita.9G158800 & 9 & 10515531 & 10521227 & 856 & 6.16 & 93049.81 & 17 & III \\
\hline Sihdz38 & Seita.9G219700 & 9 & 16264055 & 16271199 & 839 & 5.83 & 91968.72 & 17 & III \\
\hline Sihdz39 & Seita.9G248400 & 9 & 19579512 & 19581764 & 299 & 5.1 & 32729.64 & 2 & II \\
\hline Sihdz40 & Seita.9G258100 & 9 & 21176272 & 21178630 & 366 & 6.21 & 38925.15 & 1 & II \\
\hline Sihdz41 & Seita.9G301000 & 9 & 34846306 & 34848095 & 247 & 8.69 & 27267.91 & 2 & I \\
\hline \multirow{2}{*}{ Sihdz42 } & Seita.9G309800 & 9 & 35775895 & 35781959 & 871 & 5.47 & 93247.34 & 9 & IV \\
\hline & Seita.9G309800.2 & 9 & 35775895 & 35781959 & 881 & 5.43 & 94315.57 & & \\
\hline
\end{tabular}


TABLE 1: Continued.

\begin{tabular}{|c|c|c|c|c|c|c|c|c|c|}
\hline \multirow[b]{2}{*}{ NIPGR ID } & \multirow{2}{*}{$\begin{array}{l}\text { Phytozome } \\
\text { Identifier }\end{array}$} & \multicolumn{3}{|c|}{ Physical position on foxtail millet genome } & \multirow{2}{*}{$\begin{array}{c}\text { Protein } \\
\text { length (aa) }\end{array}$} & \multirow[b]{2}{*}{ PI } & \multirow{2}{*}{$\begin{array}{c}\text { Molecular } \\
\text { weight } \\
\text { (Da) }\end{array}$} & \multirow[b]{2}{*}{ Intron } & \multirow{2}{*}{$\begin{array}{c}\text { Phylogeny } \\
\text { group }\end{array}$} \\
\hline & & Chromosome & $\begin{array}{l}\text { Start position } \\
\text { (bp) }\end{array}$ & $\begin{array}{c}\text { End position } \\
\text { (bp) }\end{array}$ & & & & & \\
\hline \multirow{2}{*}{ Sihdz43 } & Seita.9G321500 & 9 & 36997661 & 37000034 & 352 & 8.93 & 37940.91 & 3 & I \\
\hline & Seita.9G321500.2 & 9 & 36997661 & 37000034 & 347 & 8.79 & 37374.21 & & \\
\hline Sihdz44 & Seita.9G481800 & 9 & 52201230 & 52203593 & 278 & 8.07 & 29367.01 & 2 & I \\
\hline Sihdz45 & Seita.9G511500 & 9 & 54329497 & 54331803 & 313 & 4.78 & 34357.78 & 2 & II \\
\hline Sihdz46 & Seita.9G524200 & 9 & 55216547 & 55218989 & 335 & 6.07 & 36879.97 & 2 & II \\
\hline Sihdz47 & Seita.9G572600 & 9 & 58368642 & 58376046 & 840 & 5.93 & 92167.29 & 17 & III \\
\hline
\end{tabular}

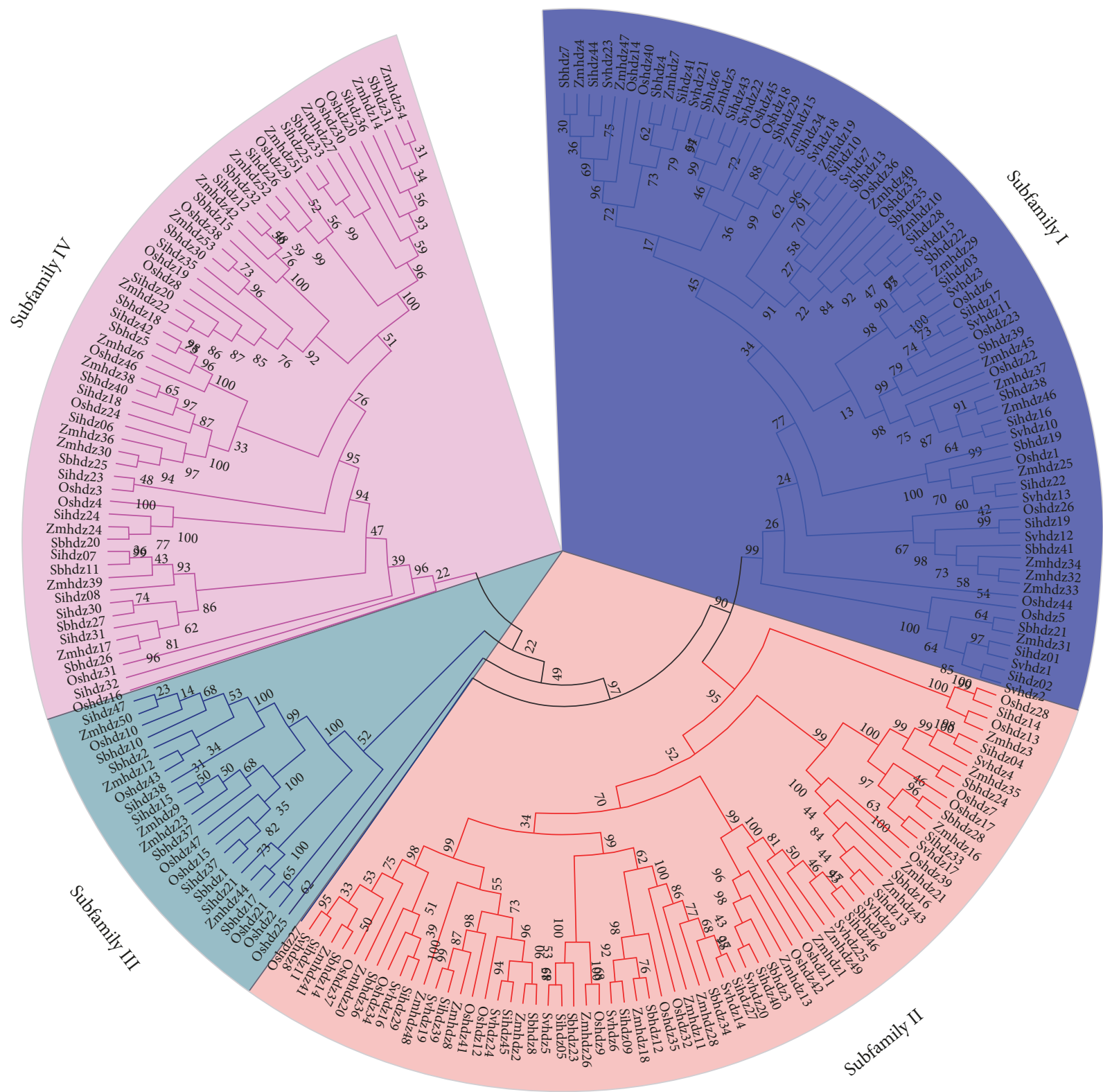

FIGURE 1: Phylogenetic tree of HD-Zip transcription factors in foxtail millet, maize, rice, green foxtail, and sorghum. The unrooted phylogenetic tree was produced with Clustal X2.0 by the neighbor-joining (NJ) method with 1000 bootstrap replications. The tree was divided into four subfamilies designated I to IV, with each HD-Zip family being indicated by a specific color. 

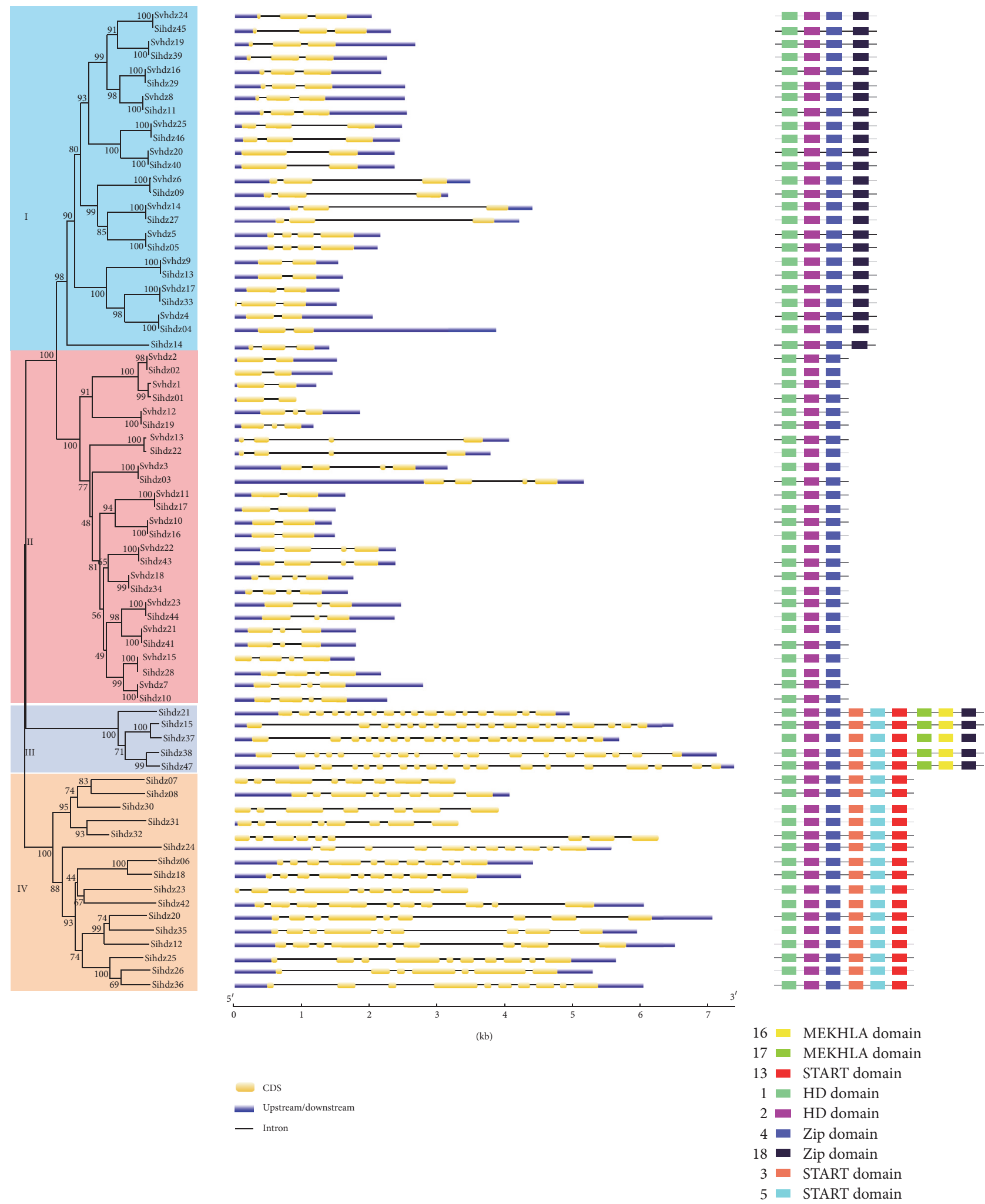

(a)

(b)

(c)

FIGURE 2: Phylogenetic relationships, structure, and motif analysis of the 47 predicted foxtail millet and 25 green foxtail HD-Zip proteins. (a) The tree was generated with the MEGA 7.0 program using the full-length amino acid sequence by the NJ method. Bootstrap values are indicated at the nodes. Each subfamily shown in the tree is marked with differently colored backgrounds. (b) Exon/intron structure of sihdz genes. Exons and introns are presented by green boxes and black lines, and untranslated regions (UTRs) are indicated by blue boxes. (c) Distribution of conserved motifs in foxtail millet HD-Zip family members. All motifs were identified using the MEME website. Differently colored boxes represent different motifs with their names on the right, and colored boxes are ordered manually according to the results of MEME analysis. The length of the box does not present the actual motif size in the protein. 
TABLE 2: Predicted motif sequence in foxtail millet HD-Zip proteins.

\begin{tabular}{lll}
\hline Motif & Width & Best possible match \\
\hline 1 & 23 & LGLEPRQVKFWFQNRRTRWKTKQ \\
2 & 29 & KKKYHRHTKEQIQELERSFKECNHPDPKQ \\
3 & 35 & YNGTIQLMYMEFYVPSPLVPTREFWFLRYCKQIED \\
4 & 15 & LREENDRLKKEIDRL \\
5 & 29 & CRRLPSGCLIQDMPNGYCKVTWVEHMEYD \\
6 & 29 & MLILQESCTDASGSYVVYAPIDVNAMNVV \\
7 & 32 & VFDYLRNEQRRGEWDIFSNGGQVQEMAHIANG \\
8 & 41 & AVHFLYRPHFQSGQAFGARRWVASLQRQCEYMAILMSSNIP \\
9 & 45 & GCLLTVAFQILVNSVPTAKLTLESVATVNSLICCTIEKIKAALKC \\
10 & 21 & NGGDPDYVPLLPSGFAIIPDG \\
11 & 41 & ENCQLRQENDKLRAENMTYKEAMRNPICPNCGGPAVLGEMS \\
12 & 25 & GKRSMMKLAQRMMRSFCDAISGFNT \\
13 & KPHGCHVEATRDCGVVIMTPTKLVEIFMDVNKWMEFFPCI \\
14 & 40 & DKPMLLEIAERTMDEFMMMATKTEPLWVP \\
15 & 29 & RKSVDDPGEPPGIVLSATTSVWLPVTPPH \\
16 & 29 & MEQGYAYLPGGVCVSGMGRHVSYEQAVAWKVLGDDSNPHCLAFMFVNWSF \\
17 & 50 & LKMFWHHSDAILCCSFKEKPMFTFANQAGLDMLETTLIALQDITLEKIFD \\
18 & 50 & EVDCEYLKRCC \\
19 & 11 & GGIICAKASMLLQNVPPAVLVRFLREHRSEWADYNIDAYSASALKANPC \\
20 & 49 & SVPEVLRPLYESPKIVAQKMTTAALRHIRQIAHETSGEVPY \\
\hline
\end{tabular}

in all 72 genes, while all of the genes had either motif 4 or motif 18 . Subfamily III and subfamily IV include motif 3 , motif 5, and motif 13 encoding the START domain. Motif 16 and motif 17, which encode the MEKHLA domain, were only found in subfamily III. In addition, it was suggested that some subfamily-specific motifs with no annotation may contribute to the diversification of the subfamily.

The Blast2GO program was used to search for the functions of HD-Zip proteins, including biological process, molecular role, and cellular component (Table S1). The results showed that sihdz proteins participate in diverse biological processes. HD-Zip I genes are usually involved in responses to abiotic stresses, ABA, and blue-light signaling. In contrast, HD-Zip II genes respond to auxins and illumination conditions. HD-Zip III and IV genes play essential roles in plant growth and development, such as meristem regulation, root development, and auxin transport. The molecular function analysis indicated that HD-Zip genes exert specific DNAbinding transcription activity. Furthermore, the cellular component analysis revealed that the products of HD-Zip genes are localized in the nucleus.

3.3. Chromosomal Location and Gene Duplication. The 47 HD-Zip genes are unevenly distributed along the nine foxtail millet chromosomes (Figure 3). The numbers of HD-Zip genes on each chromosome have a wide range. For example, chromosome 9 contains the highest number of genes (11), followed by chromosome 2 (8), while no genes are present on chromosome 8 . Some chromosomes have regions of high gene density, such as chromosome 7 and chromosome 9. Tandem and segmental duplications are usually vital to gene expansion. A previous analysis of the whole genome revealed that gene duplications occurred approximately 70 million years ago [42]. Moreover, examination of gene duplication revealed that 4 gene pairs had been tandemly duplicated, whereas 14 gene pairs had been segmentally duplicated. The analysis was also performed in green foxtail; 2 gene pairs were found to be tandemly duplicated, while 6 gene pairs were found to be segmentally duplicated (Figure S1).

The ratio of nonsynonymous to synonymous mutation rates $(\mathrm{Ka} / \mathrm{Ks})$ was used to explore the selective constraints on duplicated sihdz genes. A value of $\mathrm{Ka} / \mathrm{Ks}<1$ indicates that duplicated genes have undergone negative/purifying selection and $\mathrm{Ka} / \mathrm{Ks}=1$ indicates that genes have been in a neutral state, while $\mathrm{Ka} / \mathrm{Ks}>1$ signifies accelerated evolution with positive selection. The $\mathrm{Ka} / \mathrm{Ks}$ values of the tandem duplications were $<1$, which means that these genes had been under purifying selection pressure. For a more thorough examination of paralogous pairs in maize, we constructed an NJ tree based only on foxtail millet proteins. Genes with more than $90 \%$ homology were considered to be paralogous pairs. A total of 18 gene pairs were identified. In this case, most of the duplicated genes had $\mathrm{Ka} / \mathrm{Ks}<1$, meaning that purifying selection was vital to the functional divergence of sihdz genes. For sihdz04/sihdz33, sihdz06/sihdz18, sihdz20/sihdz35, and $\operatorname{sihd} z 34 / \operatorname{sih} d z 43$, the $\mathrm{Ka} / \mathrm{Ks}$ values were $>1$, so these genes may have played important roles in gene evolution (Table 3 ).

3.4. Promoter Analysis and Three-Dimensional Modeling of Sihdz. The cis-acting element of promoters is essential for determining tissue-specific expression and is involved in the regulation of gene expression under abiotic stress. An investigation of a region about $2000 \mathrm{bp}$ upstream of the start codon (ATG) was used here to analyze cis-acting 


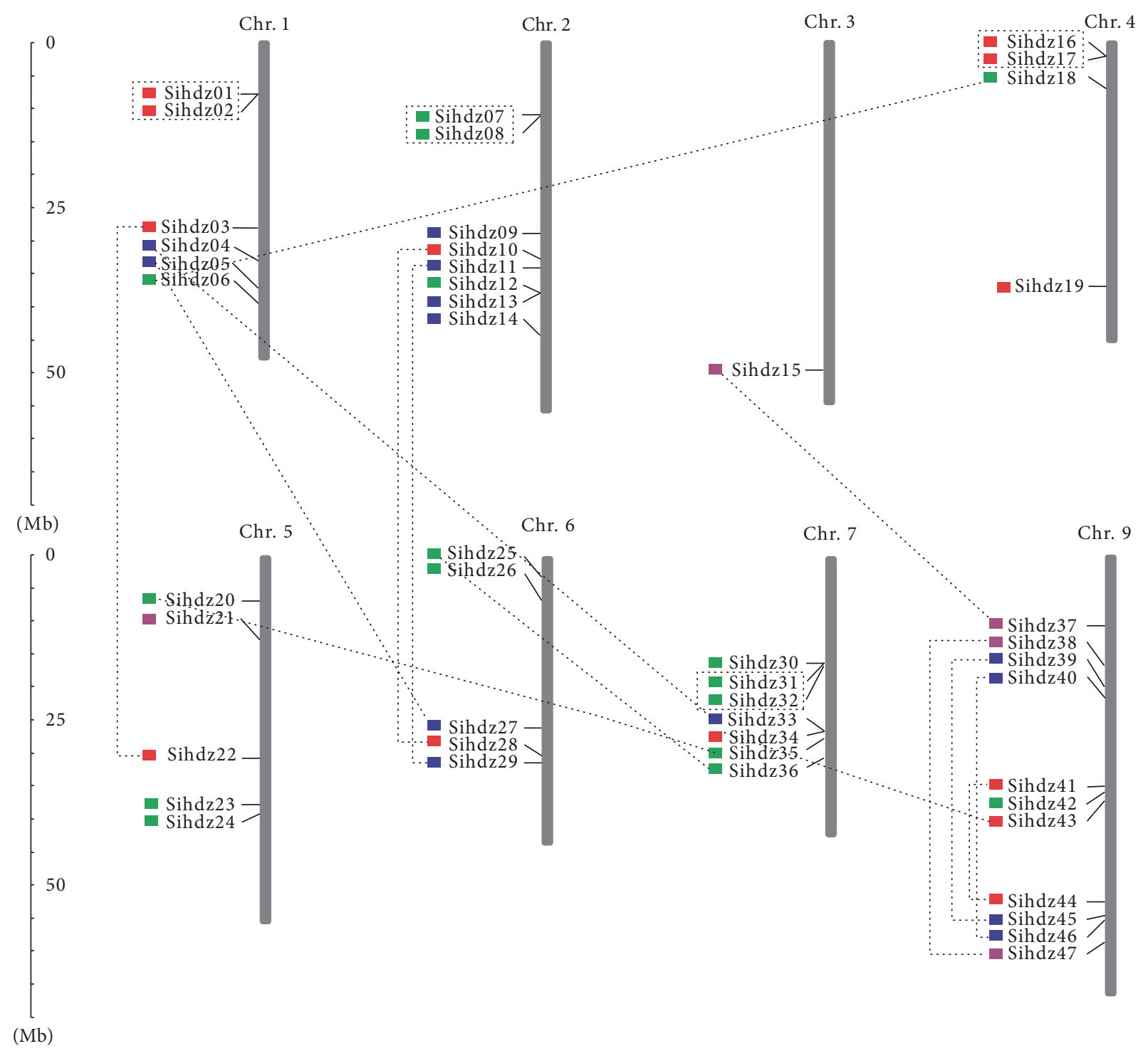

FIGURE 3: Chromosomal distribution and segmental duplication events of 47 sihdz genes in nine foxtail millet chromosomes. The 47 sihdz genes were mapped to the foxtail millet chromosomes. The genes in each subfamily are presented in different colors. The segmentally duplicated gene pairs are connected by lines.

regulatory elements. The results showed that many stressrelated elements participate in regulating gene expression. These include ABRE and motif IIb, which are involved in the response to abscisic acid; TCA element, which is involved in salicylic acid responsiveness; and MBS, which is an MYB binding site involved in drought inducibility. These elements were thought to play important roles in responses to abiotic stress, so these genes may be involved in responses to environmental conditions. The CAT box is a cis-acting regulatory element related to meristem expression. The CGTCA motif and the TGACG motif are involved in responses to MeJA stress. The GC motif is an enhancer-like element involved in anoxic-specific inducibility. The details of cis-acting elements are shown in Table S2, which provides a deeper understanding of the mechanism of stress tolerance in foxtail millet.
Three-dimensional models of all sihdz proteins were constructed using the Phyre2 server (Figure 4). By comparing homologous gene structures, genes in the different subfamilies were shown to have different features. Subfamilies I and II have simple structures, while subfamilies III and IV have complex ones. Specifically, as well as $\alpha$-helix and coil, subfamilies III and IV also have $\beta$-sheets. All of the predicted structures provide a foundation to analyze the functions of these molecules.

3.5. Orthologous Relationships of Sihdz Genes in Foxtail Millet, Maize, Rice, Sorghum, Brachypodium Distachyon, Green Foxtail, and Switchgrass. To further elucidate the relationships in foxtail millet, maize, rice, sorghum, Brachypodium distachyon, green foxtail, and switchgrass were chosen for further analysis; the orthologous genes were found using 
TABLE 3: Ka/Ks analysis and divergence between HD-Zip gene pairs in foxtail millet.

\begin{tabular}{|c|c|c|c|c|c|c|}
\hline Gene & Duplicate & Ks & $\mathrm{Ka}$ & $\mathrm{Ka} / \mathrm{Ks}$ & Purifying selection & Mya \\
\hline Sihdz01 & Sihdz02 & 0.2775 & 0.0662 & 0.238559 & Yes & 21.34615 \\
\hline Sihdz03 & Sihdz22 & 1.7425 & 0.505 & 0.289813 & Yes & 134.0385 \\
\hline Sihdz04 & Sihdz33 & 0.8217 & 1.9811 & 2.410977 & No & 63.20769 \\
\hline Sihdz05 & Sihdz27 & 0.6551 & 0.3621 & 0.55274 & Yes & 50.39231 \\
\hline Sihdz06 & Sihdz18 & 0.6382 & 1.0217 & 1.600909 & No & 49.09231 \\
\hline Sihdz07 & Sihdz08 & 1.5288 & 1.3628 & 0.891418 & Yes & 117.6 \\
\hline Sihdz10 & Sihdz28 & 0.4326 & 0.2743 & 0.634073 & Yes & 33.27692 \\
\hline Sihdz11 & Sihdz29 & 0.4042 & 0.2187 & 0.541069 & Yes & 31.09231 \\
\hline Sihdz15 & Sihdz37 & 0.6411 & 0.1003 & 0.15645 & Yes & 49.31538 \\
\hline Sihdz16 & Sihdz17 & 0.7462 & 0.3158 & 0.423211 & Yes & 57.4 \\
\hline Sihdz20 & Sihdz35 & 0.2327 & 0.2979 & 1.280189 & No & 17.9 \\
\hline Sihdz25 & Sihdz36 & 0.8552 & 0.1837 & 0.214804 & Yes & 65.78462 \\
\hline Sihdz31 & Sihdz32 & 0.3182 & 0.2121 & 0.666562 & Yes & 24.47692 \\
\hline Sihdz34 & Sihdz43 & 0.6948 & 1.3737 & 1.977116 & No & 53.44615 \\
\hline Sihdz38 & Sihdz47 & 1.0853 & 0.0731 & 0.067355 & Yes & 83.48462 \\
\hline Sihdz39 & Sihdz45 & 0.561 & 0.466 & 0.83066 & Yes & 43.15385 \\
\hline Sihdz40 & Sihdz46 & 0.46 & 0.2758 & 0.599565 & Yes & 35.38462 \\
\hline Sihdz41 & Sihdz44 & 0.9095 & 0.2868 & 0.315338 & Yes & 69.96154 \\
\hline
\end{tabular}

OrthoMCL (Figure 5). In total, 116 orthologous gene pairs were found in foxtail millet-rice, 114 were found in foxtail millet-sorghum, 74 in foxtail millet-maize, 61 in foxtail millet-green foxtail, 57 in switchgrass-millet, and 107 in Brachypodium distachyon-millet, which indicated that there were closer relationships among the monocotyledons. However, a closer relationship was found between foxtail millet and sorghum than between foxtail millet and maize, green foxtail, or switchgrass. These results are consistent with the findings of previous studies [35, 36, 38]. Some genes have no collinear genes, such as sihdz02/GRMZM2G477415 and sihdz02/Sobic.004G247500 being present in foxtail milletmaize and foxtail millet-sorghum, but sihdz02 has no paralog in rice and Brachypodium distachyon, indicating that these orthologous pairs formed after rice and Brachypodium distachyon diverged from their common ancestor. Several genes have orthologs in all six species, so these genes may have been conserved over the course of evolution.

3.6. Expression Analysis of Foxtail Millet HD-Zip Genes in Various Tissues. To obtain insight into the expression patterns of sihdz genes in various tissues, a heat map was generated using the gene expression data (Figure S2). The tissue-specific expression data used here included those from root, leaf, stem, and spica. All of the 47 sihdz genes have various expression levels in different tissues. Sihdz05 and sihdz29 were highly expressed in all four tissues, while sihdz02, sihdz24, sihdz26, sihdz34, sihdz01, sihdz51, sihdz48, sihdz40, sihdz33, sihdz32, sihdz28, sihdz25, sihdz22, sihdz20, sihdz07, and sihdz15 were rarely expressed in all tissues. Sihdz21 showed high expression in spica and low expression in leaf, indicating that it has an important role in flowering. Sihdz41 and sihdz16 have high transcript accumulation in root, stem, and spica, which may be related to gene functions.
Paralogous pairs with similar sequences exhibit divergent expression patterns. For example, sihdz38 is highly expressed in spica, while sihdz41 is highly expressed in root.

3.7. Expression Profiling of Sihdz Genes during Abiotic Stress. For adaptation to several types of abiotic stress, such as dehydration, salinity, and ABA, the expression of many stress-related genes is induced. For example, previous studies reported that the expression of HD-Zip I genes was induced by drought $[53,54]$. To gain further insight into the roles of HD-Zip genes upon exposure to drought and salinity, qRT-PCR was used to examine the expression profiles of these genes (Figure 6). The results showed that all of the HD-Zip I genes were drought- and salinity-responsive, with various expression patterns. Most of the HD-Zip I genes have the same expression profiles, such as sihdz4, sihdz5, sihdz11, sihdz13, sihdz29, sihdz45, and sihdz46 (Figures 6(a) and 6(b)). During the early phase of salinity and drought treatment, the expression of sihdz4 genes was steady, but then a drastic 7fold change was observed at $24 \mathrm{~h}$. Sihdz29 showed a more than $\sim 10$-fold change in expression during the salinity and drought treatments at $12 \mathrm{~h}$. In response to drought treatment, four genes showed the highest expression level, with a more than 12 -fold change. We also compared the expression profiles of gene pairs; some of these pairs had similar expression profiles. For example, the expression of both sihdz11 and sihdz29 was upregulated nearly fourfold and peaked at $12 \mathrm{~h}$ and then was downregulated at $24 \mathrm{~h}$.

The plant hormone abscisic acid (ABA) plays a vital role in drought and salt responses. During hormone treatment, all HD-Zip I genes were upregulated in ABA treatment (Figure $6(\mathrm{c})$ ). Four genes exhibited minor changes in expression, with a relative expression scale from 0 to 5: $\operatorname{sihdz9}$, sihdz14, sihdz27, and sihdz39. By contrast, sihdz29 and sihdz45 were 

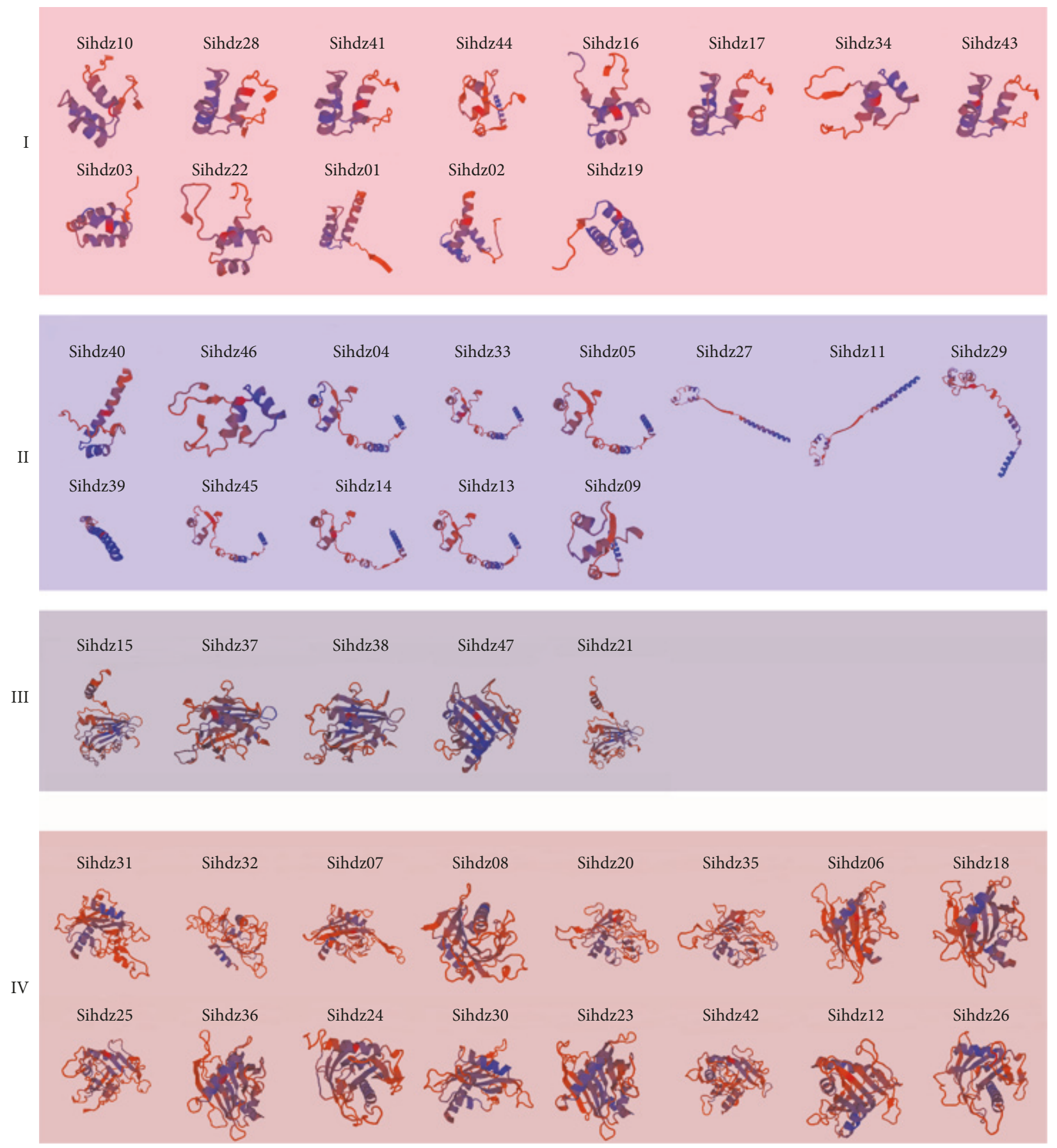

FIGURE 4: Three-dimensional modeling of sihdz proteins. The structure of sihdz proteins with a confidence level $>90 \%$ is shown and the activated sites are highlighted in blue.

clearly upregulated at all time points and peaked at $12 \mathrm{~h}$ (more than 25-fold change), while sihdz09 and sihdz46 peaked at $24 \mathrm{~h}$ (more than 15-fold change). Notably, sihdz4 had the highest expression level at $1 \mathrm{~h}$, approaching a 40 -fold change. In summary, these genes may play important roles in biotic stress responses. The expression of sihdz29 and sihdz45 was upregulated under the three treatments, so these two genes may play important roles in responses to environmental challenges.

\section{Discussion}

Setaria is a group of grasses that are important as biofuels. Many transcription factors have been performed, such as MYB transcription factor, AP2/ERF transcription factor, and the WD40 protein family $[39,55,56]$. These studies provided a foundation for evolutionary and functional characterization of transcription factors and new insight to improve the responses to environmental stimuli. The HD-Zip genes are a 

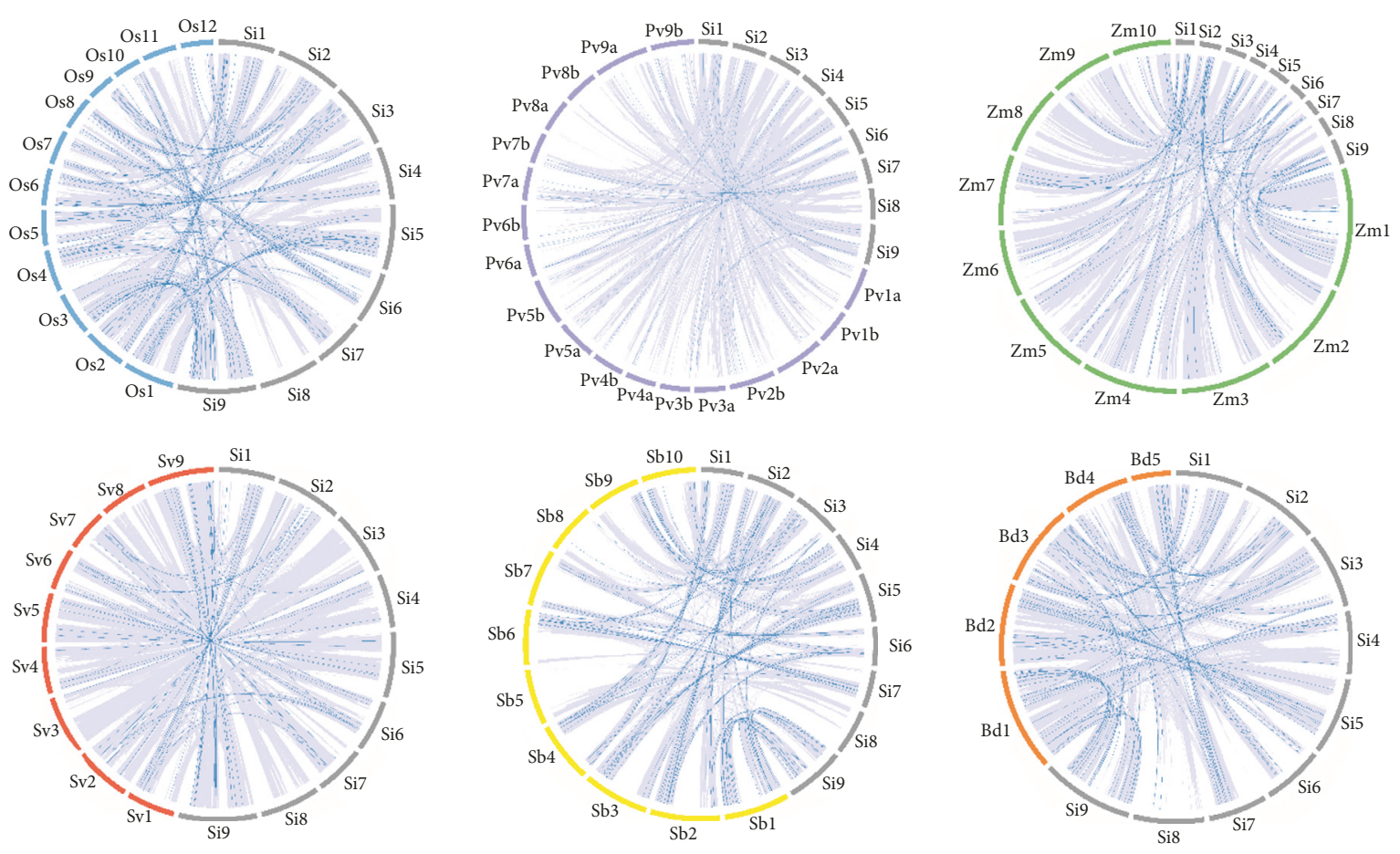

FIGURE 5: Orthologous relationships among maize, rice, sorghum, Brachypodium distachyon, green foxtail, and switchgrass. The numbers along the chromosome boxes indicate the sequence lengths in megabases. The grey line in the circle means the syntenic gene pairs in corresponding syntenic region. The blue lines represent syntenic relationships between the HD-Zip genes.

major group of transcription factors present in higher plants, which play significant roles in various biological processes during plant growth and development. The HD-Zip gene family has been researched systematically in maize and rice, but little is known about these genes in foxtail millet. We thus performed preliminary analyses of the HD-Zip gene family in foxtail millet, including its phylogeny and conserved motifs as well as three-dimensional modeling and cis-acting regulatory and expression profiling. In this study, a total of 47 nonredundant HD-Zip genes were found in the foxtail millet genome, while maize has 54 , rice has 47 , sorghum has 41 , and green foxtail has $25[12,15,57]$. The numbers of HD-Zip genes were mostly equal among these grass species, with the exception of green foxtail; this means that the HD-Zip genes have been conserved over the course of evolution. Based on their phylogenetic tree, the putative genes were clustered into four distinct subfamilies. And the gene length in different subfamilies is conserved; the length of genes in subfamilies III and IV was longer than the genes in subfamilies I and II. The exon-intron analysis also indicated that the numbers of introns in paralogous genes were conserved. Some divergence in this regard was found in $\operatorname{sih} d z 40 / \operatorname{sih} d z 46$, sihdz05/sihdz27, and sihdz31/sihdz32, indicating that a few insertions and deletions had accumulated. Subfamily I and subfamily II were found to have one to three introns, whereas subfamily III and subfamily IV had various numbers of introns, ranging from 6 to 17. Moreover, both the HD domain and the LZ domain were shown to be present in all HD-Zip genes. Subfamily III and subfamily IV contain a START domain, a highly conserved lipid/sterol binding region, while subfamily III also has a DNA-interacting PAS-related MEMKHLA domain at the C-terminus. Three-dimensional modeling analysis indicated that the genes in the same family tend to have similar protein structures. The structure and motif analysis results were consistent with the phylogenetic classification, which suggests that the classification of the genes is reliable.

Duplications such as tandem and segmental duplication events have played dominant roles in the process of genome evolution. Such gene duplications have been reported in other gene families, such as WRKY and the CCCH-type zinc finger family $[58,59]$. Our results obtained in this study indicate that segmental duplication has played important roles in HD-Zip gene expansion, especially in foxtail millet and green foxtail. The $\mathrm{Ka} / \mathrm{Ks}$ values were used to measure the course in the gene duplication and divergence [60]. Analysis of paralogous pairs showed that the values are multiple from 0.067 to 2.4. And the homology modeling indicates that the paralog genes have similar structure; maybe paralog genes have similar function. The $\mathrm{Ka} / \mathrm{Ks}$ ratios in four tandemly duplicated gene pairs ranged from 0.24 to 0.78 , suggesting that negative or purifying selection is essential in HD-Zip gene duplication. Only four segmental gene pairs are under positive selection pressure, since their $\mathrm{Ka} / \mathrm{Ks}$ ratios were estimated to be $>1$, which means that positive selection was active during the expansion of HD-Zip genes. We also estimated the Ka/Ks values of orthologous gene pairs in the genomes of four grasses. The average $\mathrm{Ka} / \mathrm{Ks}$ value peaked between foxtail 


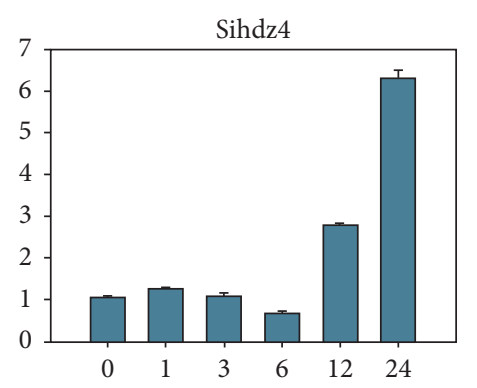

(h)

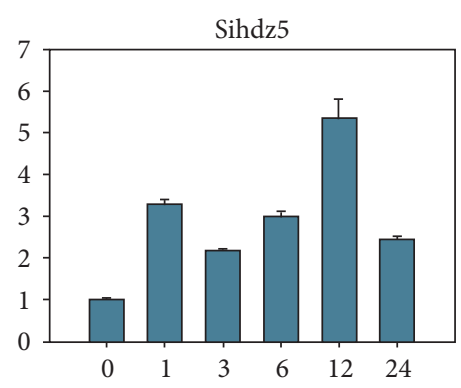

(h)

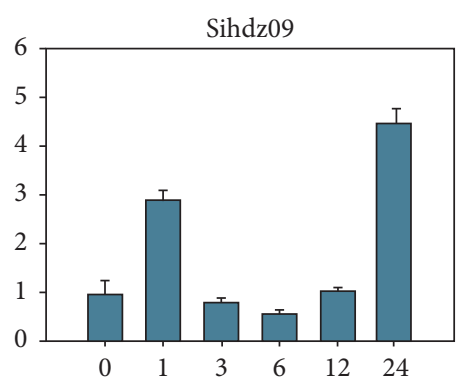

(h)

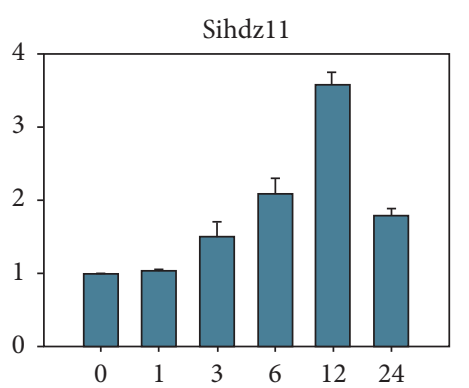

(h)

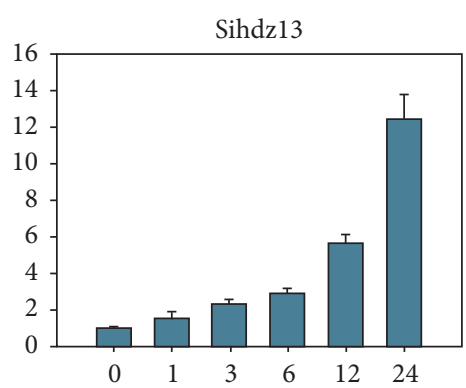

(h)

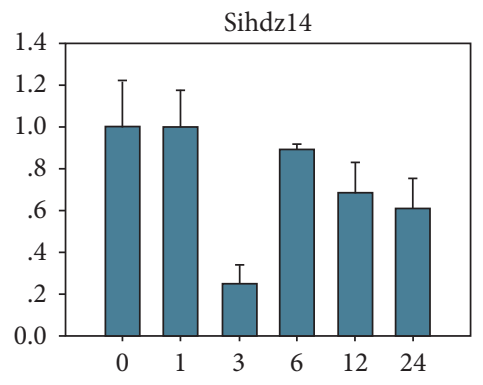

(h)

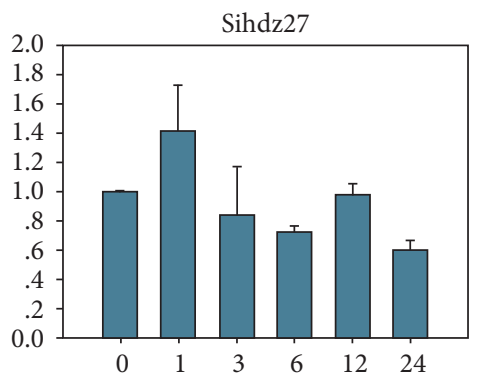

(h)

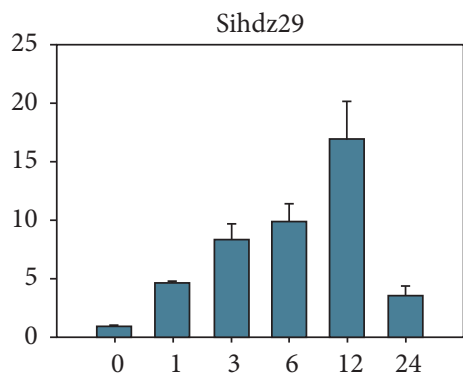

(h)

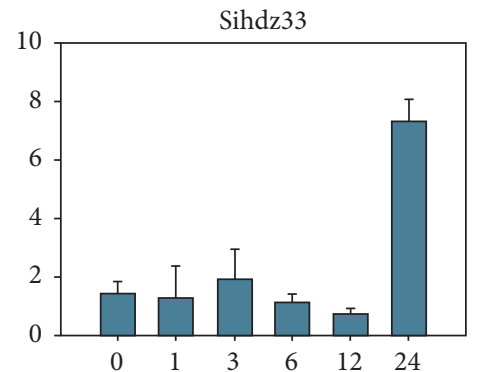

(h)

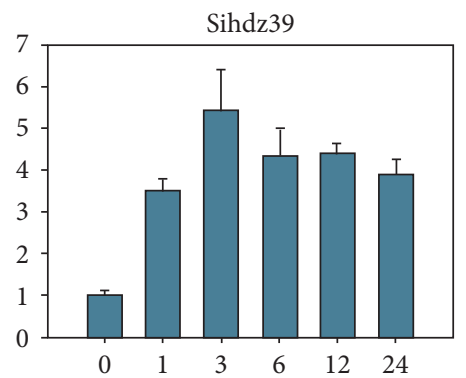

(h)

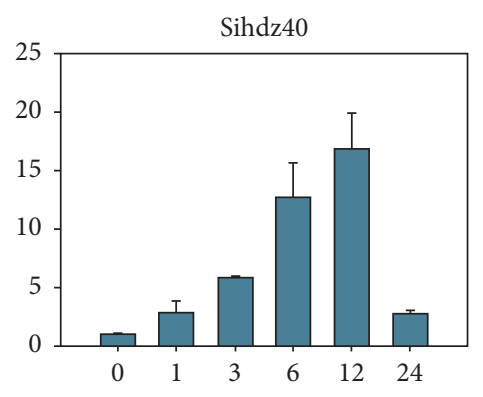

(h)

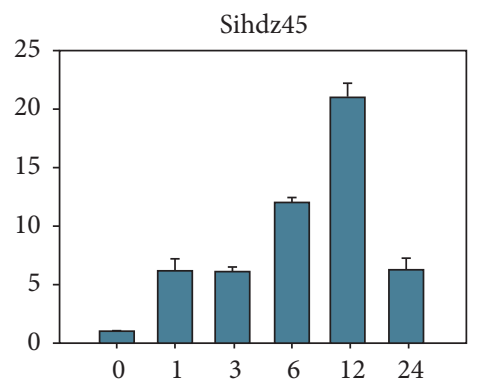

(h)

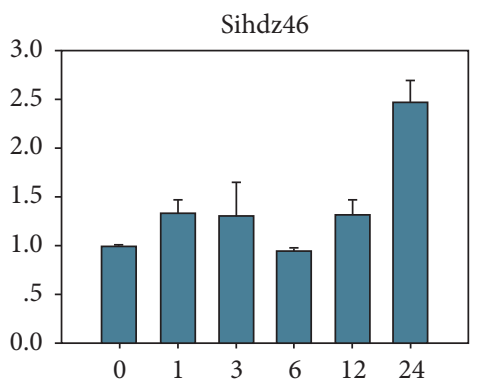

(h)

(a)

Figure 6: Continued. 


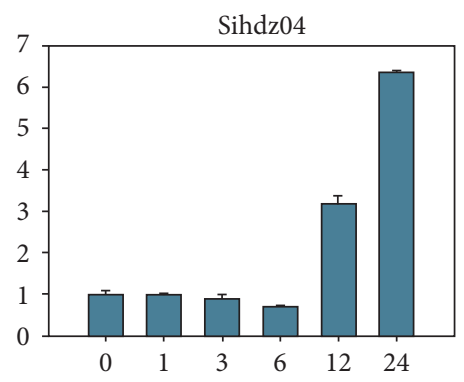

(h)

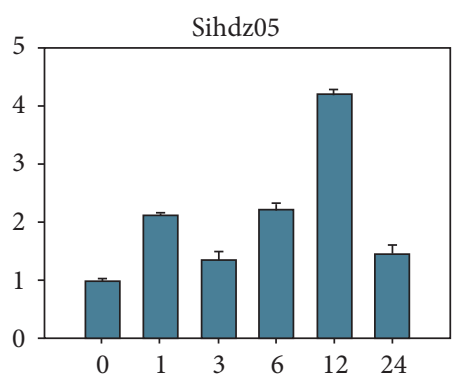

(h)

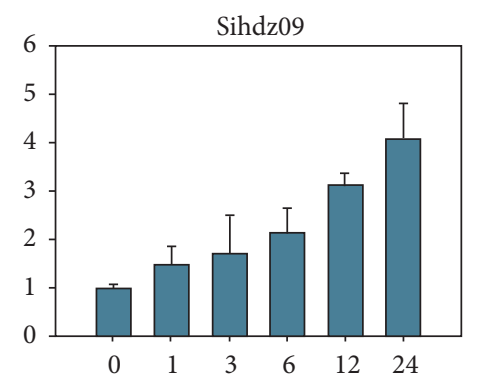

(h)

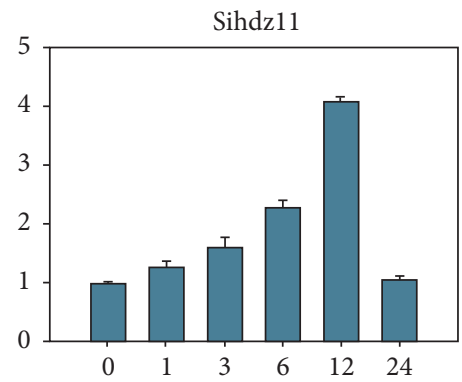

(h)

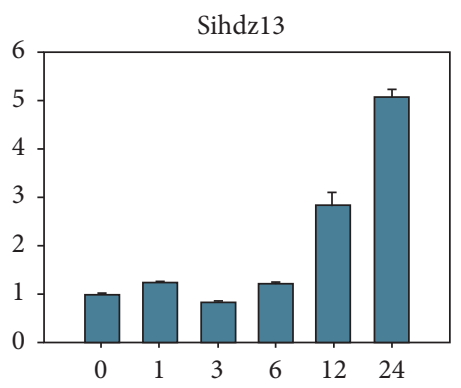

(h)

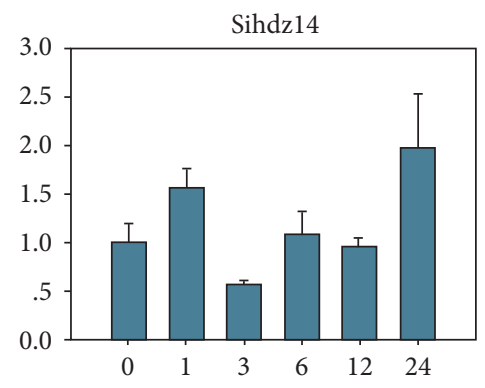

(h)

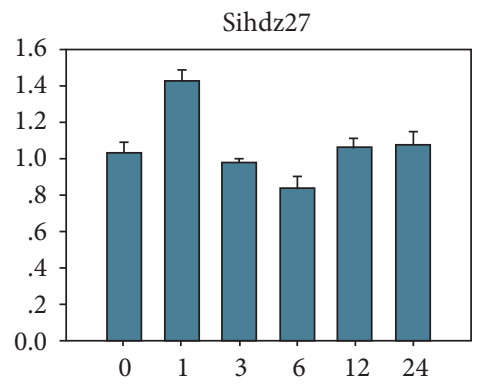

(h)

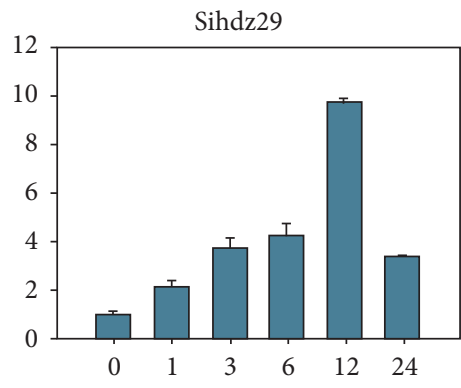

(h)

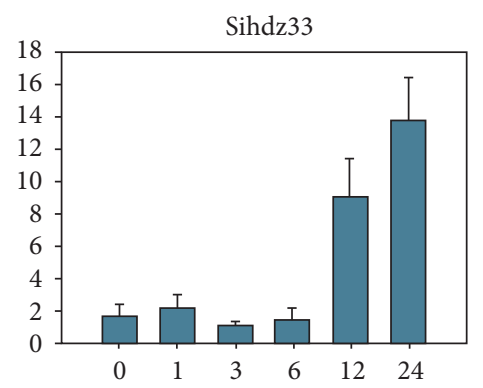

(h)

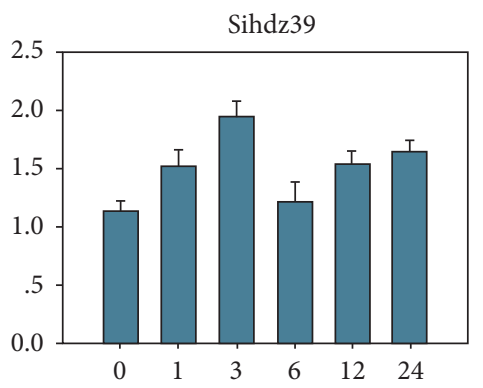

(h)

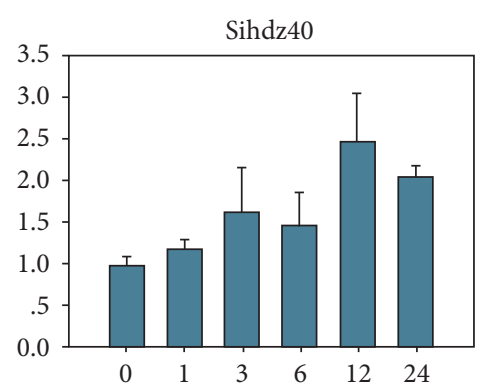

(h)

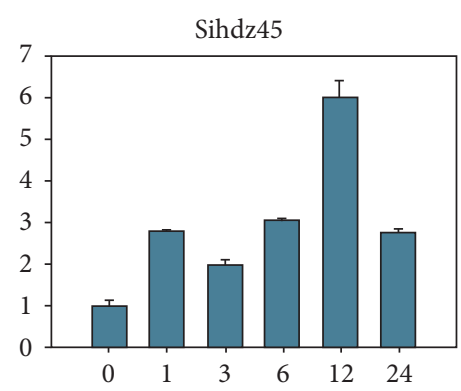

(h)

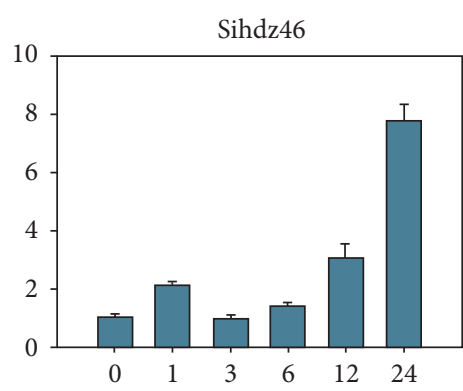

(h)

(b)

FIgURe 6: Continued. 


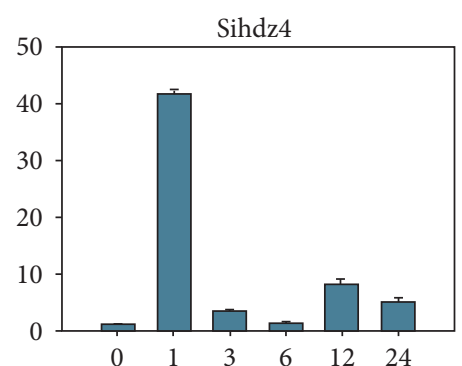

(h)

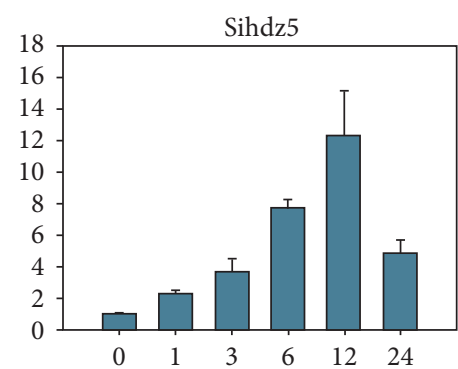

(h)

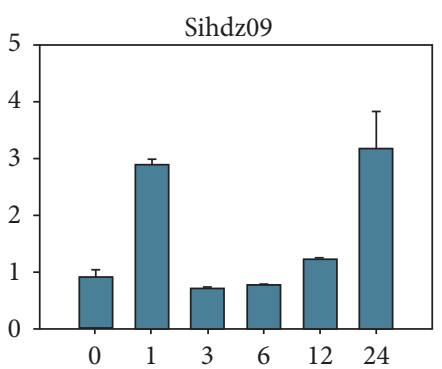

(h)

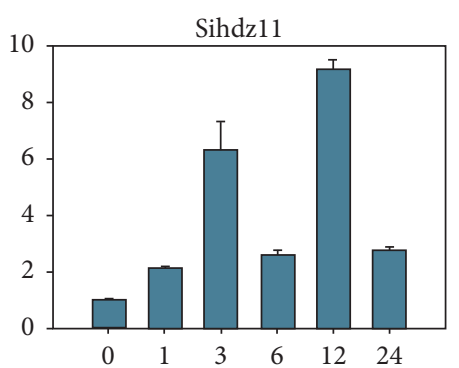

(h)

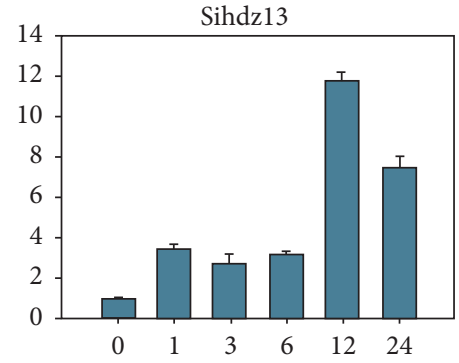

(h)

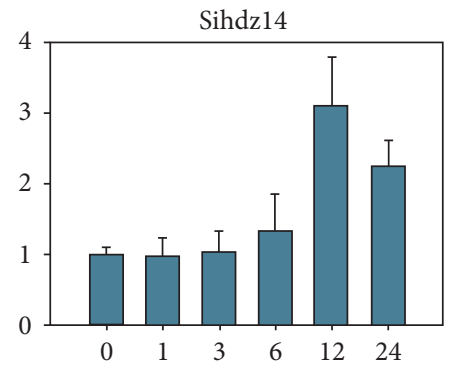

(h)

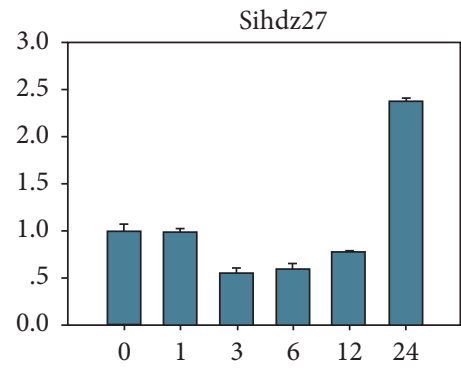

(h)

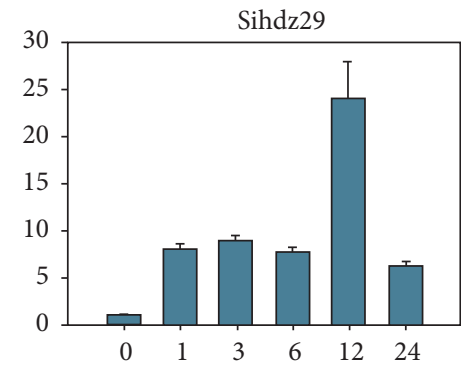

(h)

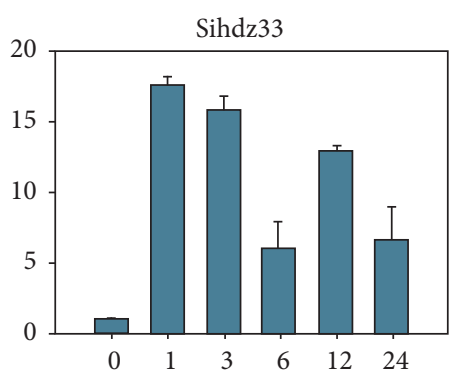

(h)

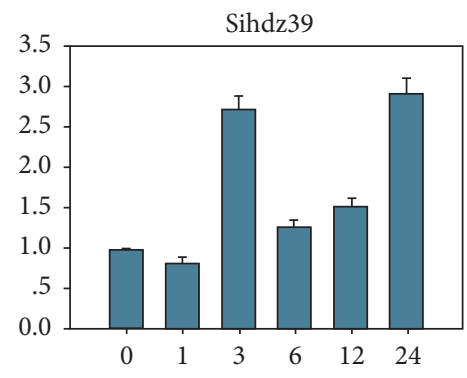

(h)

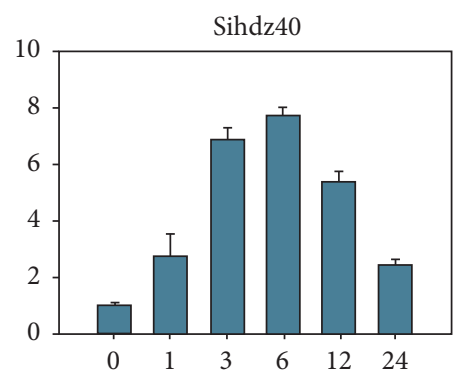

(h)

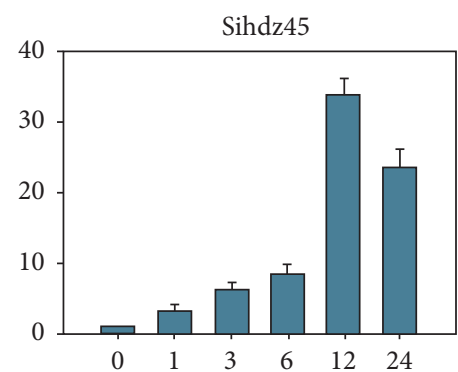

(h)

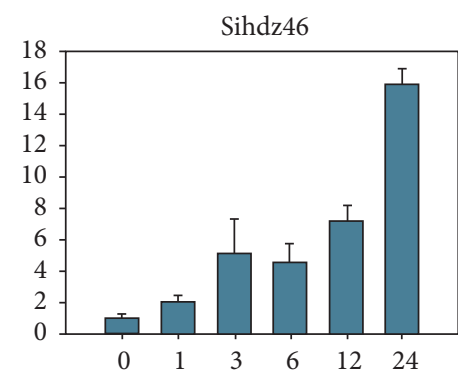

(h)

(c)

FiguRE 6: Expression profiles of 13 selected HD-Zip I genes under drought, salinity, and ABA stresses using qRT-PCR. (a) The expression profiles under PEG treatment. (b) The expression profiles under $\mathrm{NaCl}$ treatment. (c) The expression profiles under ABA treatment. The expression of each gene was calculated as a ratio relative to its expression in the control sample $(0 \mathrm{~h})$. Actin was used as an internal control to normalize the data. 


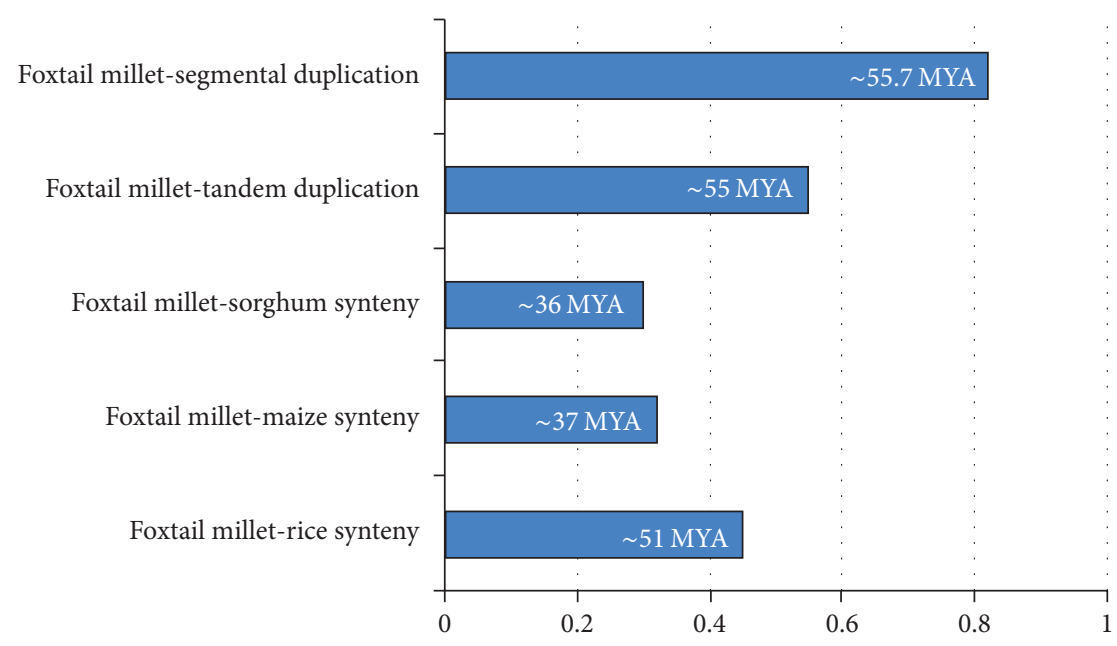

Figure 7: Time of duplication and divergence (MYA) based on synonymous substitution rate (Ks) estimated using orthologous and paralogous gene pairs between foxtail millet and sorghum, maize, or rice.

millet and rice (0.45), followed by foxtail millet and maize (0.32), and then foxtail millet and sorghum (0.30). This peak for foxtail millet and rice indicated that the HD-Zip genes diverged at an early stage, namely, around 51 MYA (Figure 7). The evolutionary analysis in foxtail millet between maize and sorghum suggests that intensive purifying selection has played a predominant role in the evolutionary process, as in other genes in foxtail millet [55]. The time of duplication in foxtail millet-maize is 37 MYA, while that in foxtail milletsorghum is $36 \mathrm{MYA}$, which is in agreement with their $\mathrm{Ka} / \mathrm{Ks}$ values $(0.32,0.30)$.

HD-Zip I genes have been demonstrated to play important roles in abiotic stress responses in rice and Arabidopsis, such as Oshox22, which was reported to participate in responses to ABA-mediated drought and salt tolerance [21]; moreover, overexpression of ATHB5 in Arabidopsis indicated that it is a positive regulator of $A B A$ responsiveness in seed germination and seedling growth [61]. However, no HDZip genes that respond to drought, salinity, and ABA have been reported in foxtail millet. As such, we investigated the expression patterns of millet HD-Zip I genes under conditions of drought, salinity, and ABA exposure. The results demonstrated that most of the genes were responsive to these three stresses. Specifically, in this study, the expression of sihdz29 and sihdz33 was strongly induced by drought, salinity, and ABA stresses, with increases of more than eightfold and tenfold. This may indicate that sihdz29 and sihdz33 regulate drought and salt tolerance through an ABA-dependent pathway. The gene pairs that have similar characteristics may have similar expression patterns, such as sihdz4/sihdz33 and $s i h d z 11 / s i h d z 29$, regardless of drought or salt tolerance. Some divergence was also found, in that sihdz40 drastically accumulated at $12 \mathrm{~h}$ (by more than 15 -fold), while sihdz46 exhibited minor expression changes under drought treatment. These findings of divergent expression patterns reveal that the HD-Zip genes may have experienced gene subfunctionalization over the course of evolution. Moreover, sihdz4, sihdz5, sihdz11, sihdz13, sihdz29, sihdz40, sihdz45, and sihdz46 have similar expression patterns in response to drought and salinity, suggesting that these genes may be involved in defense against abiotic stress. Moreover, some genes exhibited minor expression changes when exposed to PEG treatment, such as sihdz14, sihdz27, and sihdz46, and when exposed to $\mathrm{NaCl}$ treatment, such as sihdz14, sihdz27, sihdz39, and sihdz40. We conclude that HD-Zip genes might play essential roles in regulating stress-responsive pathways for adaptation to the environment.

\section{Conclusions}

We identified 47 TFs in the foxtail millet genome. Phylogenetic reconstruction showed four subfamilies in the HDZip gene family: subfamilies I, II, III, and IV. Moreover, gene structure and motif analysis showed that genes within the same subfamily were conserved. Promotor analysis showed that HD-ZIP gene was involved in abiotic stress. Expression profile analysis by qRT-PCR revealed that sih $d z 29$ and sihdz 45 may play important roles in regulating responses to multiple abiotic and biotic stresses such as $\mathrm{ABA}, \mathrm{NaCl}$, and PEG. These genes are thus potential targets for molecular breeding to improve plant tolerance. Our study also provided new insight into the evolutionary and functional characterization of HDZip genes in foxtail millet.

\section{Conflicts of Interest}

The authors declare that they have no conflicts of interest.

\section{Authors' Contributions}

Wenbo Chai and Weina Si contributed equally to this work.

\section{Acknowledgments}

The authors thank members of the National Engineering Laboratory of Crop Stress Resistance Breeding for their 
assistance in this study. This work was supported by the Key Project of National Key Research and Development Program of China (2016YFD0101002), Anhui Natural Science Foundation of the Colleges and Universities (KJ2017A147), and the National Natural Science Foundation of China (31571685 and 31771805). The authors thank Liwen Bianji, Edanz Group, China (https://www.liwenbianji.cn/ac), for editing the English text of a draft of this manuscript.

\section{Supplementary Materials}

Table S1: functions of HD-Zip proteins searched by Blast2GO. Table S2: cis-acting element of promoter. Figure S1: chromosomal distribution and segmental duplication events of $25 \mathrm{sihdz}$ genes in green foxtail. Figure S2: expression profiles of sihdz genes in different tissues. The RNA-Seq data were analyzed and a heat map was generated. Values from 0 to 7 represent low to high expression. (Supplementary Materials)

\section{References}

[1] K. Aso, M. Kato, J. A. Banks, and M. Hasebe, "Characterization of homeodomain-leucine zipper genes in the fern Ceratopteris richardii and the evolution of the homeodomain-leucine zipper gene family in vascular plants," Molecular Biology and Evolution, vol. 16, no. 4, pp. 544-552, 1999.

[2] A. E. Tron, C. W. Bertoncini, C. M. Palena, R. L. Chan, and D. H. Gonzalez, "Combinatorial interactions of two amino acids with a single base pair define target site specificity in plant dimeric homeodomain proteins," Nucleic Acids Research, vol. 29, no. 23, pp. 4866-4872, 2001.

[3] G. Sessa, G. Morelli, and I. Ruberti, “The Athb-1 and -2 HD-Zip domains homodimerize forming complexes of different DNA binding specificities," EMBO Journal, vol. 12, no. 9, pp. 35073517, 1993.

[4] J. Shaikhali, L. Norén, J. De Dios Barajas-López et al., "Redoxmediated mechanisms regulate DNA binding activity of the Ggroup of basic region leucine zipper (bZIP) transcription factors in Arabidopsis," The Journal of Biological Chemistry, vol. 287, no. 33, pp. 27510-27525, 2012.

[5] R. Hu, X. Chi, G. Chai et al., "Genome-Wide identification, evolutionary expansion, and expression profile of HomeodomainLeucine zipper gene family in poplar (populus trichocarpa)," PLoS ONE, vol. 7, no. 2, Article ID e31149, 2012.

[6] F. D. Ariel, P. A. Manavella, C. A. Dezar, and R. L. Chan, "The true story of the HD-Zip family," Trends in Plant Science, vol. 12, no. 9, pp. 419-426, 2007.

[7] S. Sen and S. Das, "Role of HD-Zip transcription factors in plant development and stress responses," International Journal of Agriculture, Environment and Biotechnology, vol. 9, no. 5, article 711, 2016.

[8] C. M. Palena, A. E. Tron, C. W. Bertoncini, D. H. Gonzalez, and R. L. Chan, "Positively charged residues at the N-terminal arm of the homeodomain are required for efficient DNA binding by homeodomain-leucine zipper proteins," Journal of Molecular Biology, vol. 308, no. 1, pp. 39-47, 2001.

[9] M. Abe, H. Katsumata, Y. Komeda, and T. Takahashi, "Regulation of shoot epidermal cell differentiation by a pair of homeodomain proteins in Arabidopsis," Development, vol. 130, no. 4, pp. 635-643, 2003.
[10] K. Schrick, D. Nguyen, W. M. Karlowski, and K. F. X. Mayer, "START lipid/sterol-binding domains are amplified in plants and are predominantly associated with homeodomain transcription factors," Genome Biology, vol. 5, no. 6, article R41, 2004.

[11] K. Ohashi-Ito, M. Kubo, T. Demura, and H. Fukuda, "Class III homeodomain leucine-zipper proteins regulate xylem cell differentiation," Plant \& Cell Physiology (PCP), vol. 46, no. 10, pp. 1646-1656, 2005.

[12] K. Mukherjee and T. R. Bürglin, "MEKHLA, a novel domain with similarity to PAS domains, is fused to plant homeodomainleucine zipper III proteins," Plant Physiology, vol. 140, no. 4, pp. 1142-1150, 2006.

[13] M. Javelle, C. Klein-Cosson, V. Vernoud et al., "Genomewide characterization of the HD-ZIP IV transcription factor family in maize: Preferential expression in the epidermis," Plant Physiology, vol. 157, no. 2, pp. 790-803, 2011.

[14] T. Huang, Y. Harrar, C. Lin et al., "Arabidopsis KANADI1 acts as a transcriptional repressor by interacting with a specific cis-element and regulates auxin biosynthesis, transport, and signaling in opposition to HD-ZIPIII factors," The Plant Cell, vol. 26, no. 1, pp. 246-262, 2014.

[15] Y. Zhao, Y. Zhou, H. Jiang et al., "Systematic analysis of sequences and expression patterns of drought-responsive members of the HD-Zip gene family in maize," PLOS ONE, vol. 6, no. 12, Article ID e28488, 2011.

[16] A. Agalou, S. Purwantomo, E. Övernäs et al., "A genomewide survey of HD-Zip genes in rice and analysis of droughtresponsive family members," Plant Molecular Biology, vol. 66, no. 1-2, pp. 87-103, 2008.

[17] S.-G. Li, W.-F. Li, S.-Y. Han, W.-H. Yang, and L.-W. Qi, "Stagespecific regulation of four HD-ZIP III transcription factors during polar pattern formation in Larix leptolepis somatic embryos," Gene, vol. 522, no. 2, pp. 177-183, 2013.

[18] W. Liu, R. Fu, Q. Li, J. Li, L. Wang, and Z. Ren, "Genome-wide identification and expression profile of homeodomain-leucine zipper Class I gene family in Cucumis sativus," Gene, vol. 531, no. 2, pp. 279-287, 2013.

[19] X. Chen, Z. Chen, H. Zhao, Y. Zhao, B. Cheng, and Y. Xiang, "Genome-wide analysis of soybean HD-Zip gene family and expression profiling under salinity and drought treatments," PLoS ONE, vol. 9, no. 2, Article ID e87156, 2014.

[20] A. Song, P. Li, J. Xin et al., "Transcriptome-wide survey and expression profile analysis of putative chrysanthemum HD-Zip I and II Genes," Gene, vol. 7, no. 5, article no. 19, 2016.

[21] S. Zhang, I. Haider, W. Kohlen et al., "Function of the HD-Zip I gene Oshox22 in ABA-mediated drought and salt tolerances in rice," Plant Molecular Biology, vol. 80, no. 6, pp. 571-585, 2012.

[22] F. Ariel, A. Diet, M. Verdenaud et al., "Environmental regulation of lateral root emergence in Medicago truncatula requires the HD-zip i transcription factor HB1 w," The Plant Cell, vol. 22, no. 7, pp. 2171-2183, 2010.

[23] Y. Zhao, Q. Ma, X. Jin et al., "A novel maize homeodomainleucine zipper (HD-Zip) i gene, Zmhdz10, positively regulates drought and salt tolerance in both rice and arabidopsis," Plant \& Cell Physiology (PCP), vol. 55, no. 6, pp. 1142-1156, 2014.

[24] J. C. Harris, M. Hrmova, S. Lopato, and P. Langridge, "Modulation of plant growth by HD-Zip class I and II transcription factors in response to environmental stimuli," New Phytologist, vol. 190, no. 4, pp. 823-837, 2011.

[25] L. Turchi, M. Carabelli, V. Ruzza et al., "Arabidopsis HD-Zip II transcription factors control apical embryo development and 
meristem function," Development, vol. 140, no. 10, pp. 2118-2129, 2013.

[26] J. Boutorrent, M. Salla-Martret, R. Brandt et al., "ATHB4 and HAT3, two class II HD-ZIP transcription factors, control leaf development in Arabidopsis," Plant Signaling and Behavior, vol. 7, no. 11, article 1382, 2012.

[27] C. A. Dezar, J. I. Giacomelli, P. A. Manavella et al., "HAHB10, a sunflower HD-Zip II transcription factor, participates in the induction of flowering and in the control of phytohormonemediated responses to biotic stress," Journal of Experimental Botany, vol. 62, no. 3, pp. 1061-1076, 2011.

[28] C. L. Côté, F. Boileau, V. Roy et al., "Gene family structure, expression and functional analysis of HD-Zip III genes in angiosperm and gymnosperm forest trees," BMC Plant Biology, vol. 10, article no. 273, 2010.

[29] M. Ilegems, V. Douet, M. Meylan-Bettex et al., "Interplay of auxin, KANADI and Class III HD-ZIP transcription factors in vascular tissue formation," Development, vol. 137, no. 6, pp. 975984, 2010.

[30] K. Ohashi-Ito and H. Fukuda, "HD-Zip III Homeobox Genes that Include a Novel Member, ZeHB-13 (Zinnia)/ATHB-15 (Arabidopsis), are Involved in Procambium and Xylem Cell Differentiation," Plant \& Cell Physiology (PCP), vol. 44, no. 12, pp. 1350-1358, 2003.

[31] D. Otsuga, B. DeGuzman, M. J. Prigge, G. N. Drews, and S. E. Clark, "REVOLUTA regulates meristem initiation at lateral positions," The Plant Journal, vol. 25, no. 2, pp. 223-236, 2001.

[32] A. Khosla, J. M. Paper, A. P. Boehler, A. M. Bradley, T. R. Neumann, and K. Schrick, "HD-Zip proteins GL2 and HDG11 have redundant functions in Arabidopsis trichomes, and GL2 activates a positive feedback loop via MYB23," The Plant Cell, vol. 26, no. 5, pp. 2184-2200, 2014.

[33] M. Javelle, V. Vernoud, N. Depège-Fargeix et al., "Overexpression of the epidermis-specific homeodomain-leucine zipper IV transcription factor OUTER CELL LAYER1 in maize identifies target genes involved in lipid metabolism and cuticle biosynthesis," Plant Physiology, vol. 154, no. 1, pp. 273-286, 2010.

[34] V. Vernoud, G. Laigle, F. Rozier, R. B. Meeley, P. Perez, and P. M. Rogowsky, "The HD-ZIP IV transcription factor OCL4 is necessary for trichome patterning and anther development in maize," The Plant Journal, vol. 59, no. 6, pp. 883-894, 2009.

[35] M. Muthamilarasan and M. Prasad, "Advances in Setaria genomics for genetic improvement of cereals and bioenergy grasses," Theoretical \& Applied Genetics, vol. 128, no. 1, pp. 1-14, 2015.

[36] X. Diao, J. Schnable, J. L. Bennetzen, and L. I. Jiayang, "Initiation of Setaria as a model plant," Frontiers of Agricultural Science \& Engineering, vol. 1, no. 1, article 16, 2014.

[37] C. Lata, S. Gupta, and M. Prasad, "Foxtail millet: A model crop for genetic and genomic studies in bioenergy grasses," Critical Reviews in Biotechnology, vol. 33, no. 3, pp. 328-343, 2013.

[38] K. Kikuchi, C. Shyu, and T. P. Brutnell, "Genetics and Genomics of Setaria," Plant Genetics \& Genomics Crops \& Models, 2017.

[39] A. K. Mishra, M. Muthamilarasan, Y. Khan, S. K. Parida, and M. Prasad, "Genome-wide investigation and expression analyses of WD40 protein family in the model plant foxtail millet (Setaria italica L.)," PLoS ONE, vol. 9, no. 1, Article ID e86852, 2014.

[40] P. Li and T. P. Brutnell, "Setaria viridis and Setaria italica, model genetic systems for the Panicoid grasses," Journal of Experimental Botany, vol. 62, no. 9, pp. 3031-3037, 2011.
[41] J. L. Bennetzen, J. Schmutz, H. Wang et al., "Reference genome sequence of the model plant Setaria," Nature Biotechnology, vol. 30, no. 6, pp. 555-561, 2012.

[42] J. Wang, G. Zhang, X. Liu et al., "Genome sequence of foxtail millet (Setaria italica) provides insights into grass evolution and biofuel potential," Nature Biotechnology, vol. 30, no. 6, pp. 549$554,2012$.

[43] R. D. Finn, A. Bateman, J. Clements et al., "Pfam: the protein families database," Nucleic Acids Research, vol. 42, pp. D222D230, 2014.

[44] I. Letunic, T. Doerks, and P. Bork, "SMART 7: recent updates to the protein domain annotation resource," Nucleic Acids Research, vol. 40, no. 1, pp. D302-D305, 2012.

[45] T. L. Bailey, M. Boden, F. A. Buske et al., "MEME SUITE: tools for motif discovery and searching," Nucleic Acids Research, vol. 37, no. 2, pp. W202-W208, 2009.

[46] N. Saitou and M. Nei, "The neighbor-joining method: a new method for reconstructing phylogenetic trees," Molecular Biology and Evolution, vol. 4, no. 4, pp. 406-425, 1987.

[47] A. Conesa, S. Götz, J. M. García-Gómez, J. Terol, M. Talón, and M. Robles, "Blast2GO: a universal tool for annotation, visualization and analysis in functional genomics research," Bioinformatics, vol. 21, no. 18, pp. 3674-3676, 2005.

[48] K. Higo, Y. Ugawa, M. Iwamoto, and H. Higo, "PLACE: A database of plant cis-acting regulatory DNA elements," Nucleic Acids Research, vol. 26, no. 1, pp. 358-359, 1998.

[49] L. A. Kelley, S. Mezulis, C. M. Yates, M. N. Wass, and M. J. E. Sternberg, "The Phyre2 web portal for protein modeling, prediction and analysis," Nature Protocols, vol. 10, no. 6, pp. 845858, 2015.

[50] L. Li, C. J. Stoeckert Jr., and D. S. Roos, "OrthoMCL: identification of ortholog groups for eukaryotic genomes," Genome Research, vol. 13, no. 9, pp. 2178-2189, 2003.

[51] M. Krzywinski, J. Schein, I. Birol et al., "Circos: An information aesthetic for comparative genomics," Genome Research, vol. 19, no. 9, pp. 1639-1645, 2009.

[52] X. M. Huang, S. T. Zhang, C. Yang, and F. Wang, "Comparison of Different Methods for Total RNA Extraction from Maize Pollen," Journal of Henan Agricultural Sciences, 2009.

[53] C. A. Dezar, G. M. Gago, D. H. González, and R. L. Chan, "Hahb-4, a sunflower homeobox-leucine zipper gene, is a developmental regulator and confers drought tolerance to Arabidopsis thaliana plants," Transgenic Research, vol. 14, no. 4, pp. 429-440, 2005.

[54] E. Söderman, J. Mattsson, and P. Engström, “The Arabidopsis homeobox gene ATHB-7 is induced by water deficit and by abscisic acid," The Plant Journal, vol. 10, no. 2, pp. 375-381, 1996.

[55] M. Muthamilarasan, R. Khandelwal, C. B. Yadav, V. S. Bonthala, Y. Khan, and M. Prasad, "Identification and Molecular Characterization of MYB Transcription Factor Superfamily in C4 Model Plant Foxtail Millet (Setaria italica L.)," PLoS ONE, vol. 9, no. 10, Article ID e109920, 2014.

[56] C. Lata, A. K. Mishra, M. Muthamilarasan, V. S. Bonthala, Y. Khan, and M. Prasad, "Genome-wide investigation and expression profiling of AP2/ERF transcription factor superfamily in foxtail millet (Setaria italica L.)," PLoS ONE, vol. 9, no. 11, Article ID e113092, 2014.

[57] D. Chen, Z. Chen, M. Wu et al., "Genome-Wide Identification and Expression Analysis of the HD-Zip Gene Family in Moso Bamboo (Phyllostachys edulis)," Journal of Plant Growth Regulation, pp. 1-15, 2016. 
[58] R. Goel, A. Pandey, P. K. Trivedi, and M. H. Asif, "Genomewide analysis of the musa WRKY gene family: Evolution and differential expression during development and stress," Frontiers in Plant Science, vol. 7, no. 2016, article no. 299, 2016.

[59] X. Peng, Y. Zhao, J. Cao et al., "CCCH-type zinc finger family in maize: genome-wide identification, classification and expression profiling under abscisic acid and drought treatments," PLOS ONE, vol. 7, no. 7, Article ID e40120, 2012.

[60] A. Nekrutenko, K. D. Makova, and W.-H. Li, "The KA/KS ratio test for assessing the protein-coding potential of genomic regions: an empirical and simulation study," Genome Research, vol. 12, no. 1, pp. 198-202, 2002.

[61] H. Johannesson, Y. Wang, J. Hanson, and P. Engström, “The Arabidopsis thaliana homeobox gene ATHB5 is a potential regulator of abscisic acid responsiveness in developing seedlings," Plant Molecular Biology, vol. 51, no. 5, pp. 719-729, 2003. 


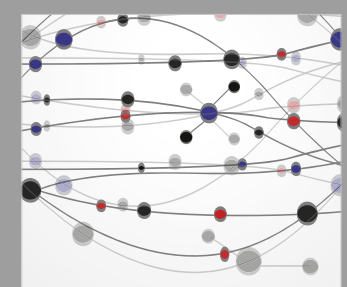

The Scientific World Journal
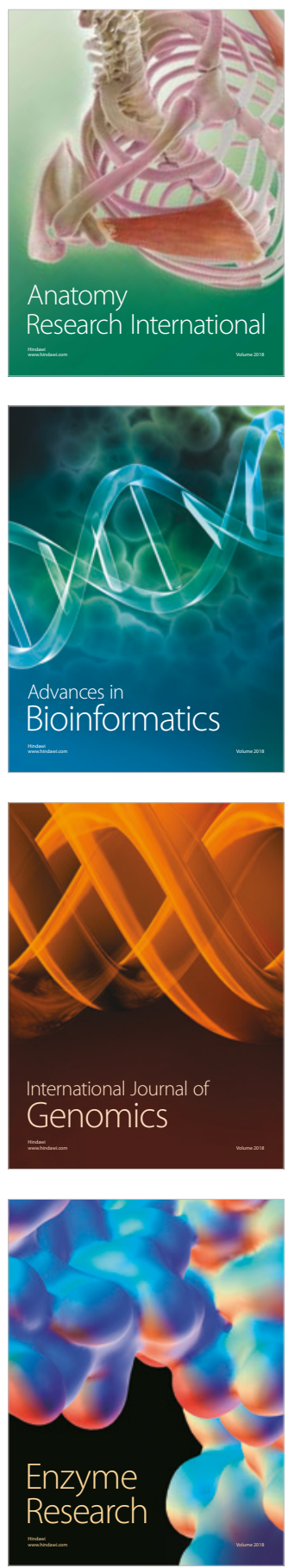
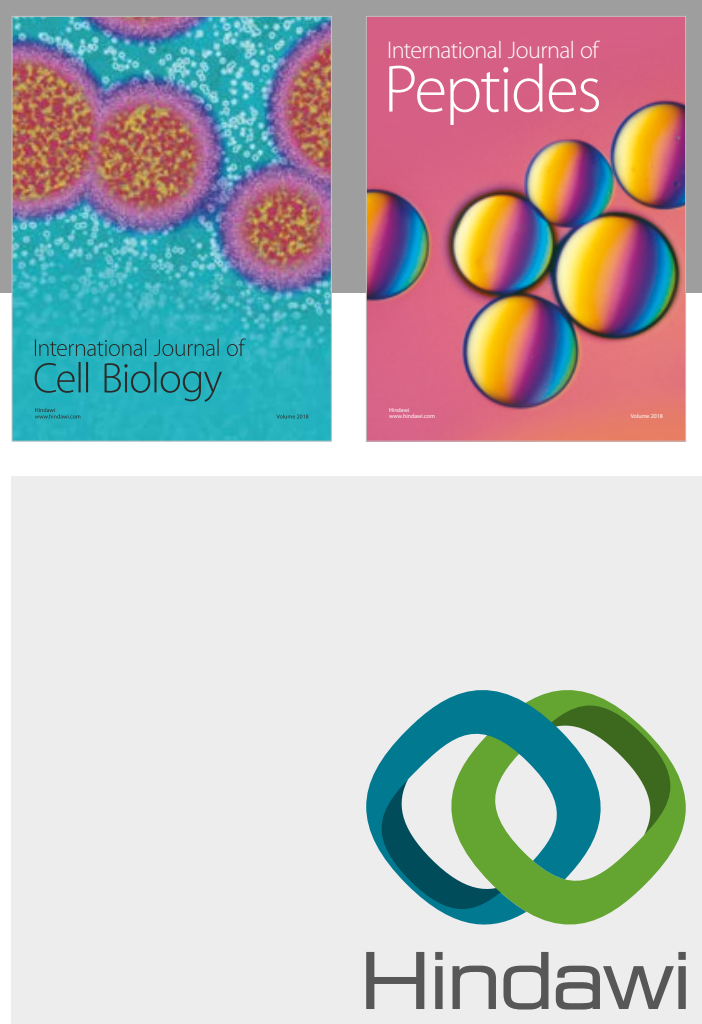

Submit your manuscripts at

www.hindawi.com
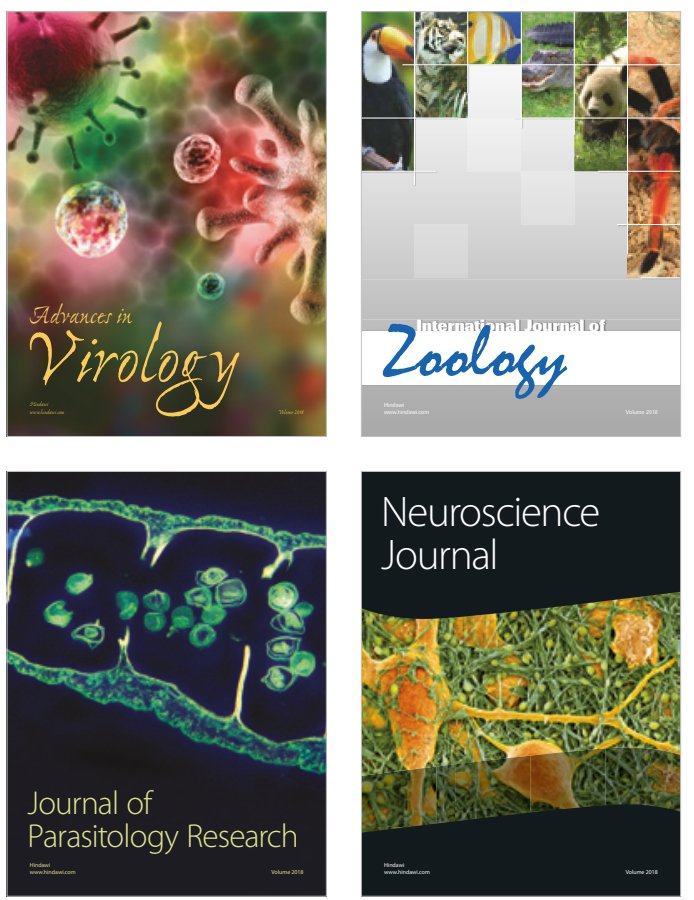
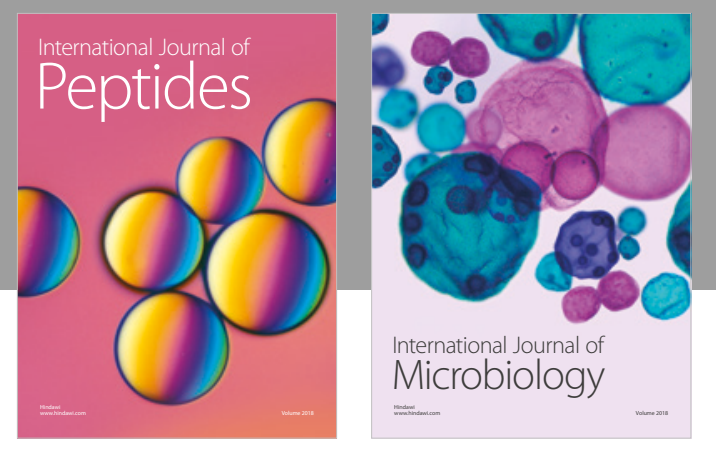

nternational Journal of Microbiology
Journal of
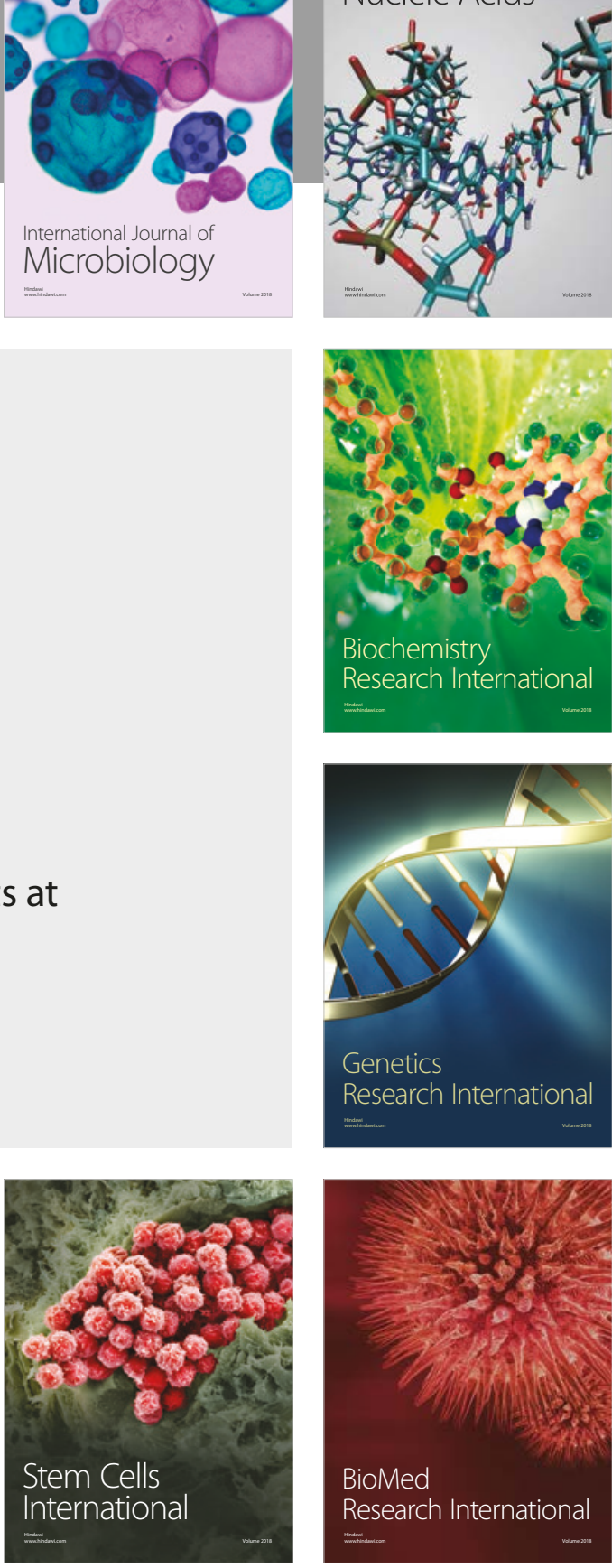
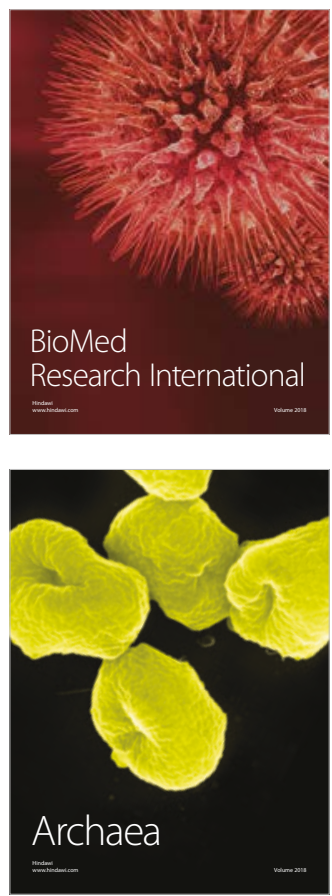